\title{
Single-Chain Polymer Nanoparticles via Non-Covalent and Dynamic Covalent Bonds
}

\author{
Ana Sanchez-Sanchez and José A. Pomposo*
}

A. Sanchez-Sanchez, Prof. J. A. Pomposo

Centro de Física de Materiales (CSIC, UPV/EHU) - Materials Physics Center. Paseo Manuel de Lardizabal 5, 20018 San Sebastián, Spain

Departamento de Ciencia y Tecnología de Polímeros, Universidad del País Vasco

(UPV/EHU). Paseo Manuel de Lardizabal 3, 20018 San Sebastián, Spain

E-mail: Josetxo.pomposo@ehu.es

Prof. J. A. Pomposo

IKERBASQUE - Basque Foundation for Science. Alameda Urquijo 36, 48011 Bilbao, Spain

Keywords: Supramolecular; single-chain; nanoparticles; synthesis; self-assembly; catalysis; nanomedicine.

The added-value behind reversible bonds is the possibility to develop responsive structurally dynamic materials which can adapt their constitution and, hence, properties to external stimuli. In recent years, much effort has been devoted to the design and synthesis of single-chain polymer nanoparticles (SCNPs) that can be reversibly assembled / disassembled in response to external environmental changes or even that can adapt their structure through simple exchange reactions. Here, the evolution of the relatively new field of responsive SCNPs constructed via non-covalent, or supramolecular, interactions and "dynamic" covalent bonds is described in detail. The main reversible bonds commonly used in supramolecular polymer chemistry and dynamic covalent materials are summarized. A detailed description of the current reported routes to reversible unimolecular polymer nanoparticles is provided. Finally, future prospects of SCNPs constructed via supramolecular interactions and dynamic bonds are detailed.

\section{Introduction}

Complexity and molecular function of natural biomacromolecules such as proteins which are present at a concentration as high as $200 \mathrm{mg} / \mathrm{ml}$ in cells can be attributed to Nature's efficient use of non-covalent bonds (e.g., hydrogen bonding, hydrophobic interactions, $\pi-\pi$ stacking, metal coordination) to form sophisticated functional architectures, as a result of millions of years of evolution. Recently, inspired by Nature's functional nanomachines and significant 
advances in macromolecular synthesis and characterization techniques, the concept of singlechain technologies has been introduced by Lutz, Sawamoto and coworkers ${ }^{[1]}$ aimed at the construction of devices based on discrete macromolecules with carefully engineered primary and secondary structures for microelectronics, photovoltaics, catalysis and biotechnology applications. In this sense, folding of individual polymer chains through non-covalent and dynamic covalent bonds is a promising pathway towards artificial, adaptative and smart single-chain polymer nanodevices.

Non-covalent bonds have fascinated scientists from different disciplines since the excellent work about hydrogen bonding by Linus Pauling in the 1930 's. ${ }^{[2]}$ More recently, the “supramolecular chemistry” concept introduced by Jean Marie Lehn ${ }^{[3]}$ opened the door to exploiting non-covalent bonds for the construction of a variety of advanced artificial architectures, including supramolecular polymers. A canonical example of "non-covalent chemistry beyond the molecule” is main-chain supramolecular polymers constructed via selfassembly of small molecular fragments that are held together by strong and directional noncovalent bonds (e.g., hydrogen bonding interactions). A clear demonstration that main-chain supramolecular polymers are susceptible to external environment changes is that their molecular weights often depend on solvent polarity and concentration. This is a consequence of the effect of many factors (i.e., temperature, pressure, concentration, etc.) on the strength of non-covalent bonds and, consequently, on the equilibrium between bonded and non-bonded or free species. Non-covalent or supramolecular interactions have been used to generate complex architectures involving non-covalent side-chain polymers, ${ }^{[4]}$ multi-block supramolecular polymers $^{[5]}$ and multi-arm self-assembled stars. ${ }^{[6]}$

Complementary to the concept of responsive non-covalent interactions is that of reversible or "dynamic" covalent chemistry. ${ }^{[7]}$ The dynamic covalent bond, having the nature of a robust covalent bond, can be break and reform only when specific external factors are 
present (e.g., catalysts). Otherwise, the reversible covalent bond is not susceptible to changes in concentration, solvent or, in certain cases, temperature. A classical example of dynamic covalent chemistry involves the disulfide bond that responds to $\mathrm{pH}$, light and redox conditions. In Nature, disulfide bond formation contributes to the folding and stabilization of proteins, usually proteins secreted to the extracellular medium, but also thiol-disulfide exchange reactions are involved in signal transduction, thiol protection, and redox homeostasis regulation. ${ }^{[8]}$ A variety of structurally dynamic polymers has been demonstrated based on alkoxyamine bonds, ${ }^{[9]}$ Diels-Alders adducts $^{[10]}$ or hydrazone chemistry. ${ }^{[11]}$ Moreover, reversible covalent chemistry has been applied with success in the development of dynamic combinatorial libraries $^{[12]}$ and for drug discovery ${ }^{[13]}$ and controlled delivery of fragrances. ${ }^{[14]}$

The added-value behind reversible (non-covalent and covalent) bonds is the possibility to develop responsive structurally dynamic materials which can adapt their constitution, and hence properties, to external stimuli. ${ }^{[15]}$ Despite the availability of several recent reviews ${ }^{[16-21]}$ covering the use of reversible bonds for the construction of supramolecular polymeric materials, we found that none of them focuses on recent advances in SCNP construction through "reversible" bonds. Only a feature article by Altintas and Barner-Kowollik ${ }^{[22]}$ describes, among other topics, single chain folding by non-covalent interactions -up to 2011-. A recent review by Pomposo and co-workers ${ }^{[23]}$ focus exclusively in "click" chemistry routes to irreversible SCNPs. Herein, we cover the state of the art in SCNP formation through noncovalent and dynamic covalent bonds from the first report of non-covalent cross-linked single-chain nanoparticles by Hawker, Kim and colleagues ${ }^{[24]}$ up to middle of 2013. Consequently, this progress report complements the recent review about synthetic routes to irreversible SCNPs. ${ }^{[23]}$ In next section we summarize the main non-covalent interactions often used in supramolecular polymer chemistry, classified as a function of interaction strength, as well as the most important dynamic covalent chemistries which are currently available. Later, 
we describe the most important routes to reversible SCNPs via non-covalent and dynamic covalent bonds. The final part of this Progress Report presents an overview of potential applications of reversible SCNPs with special emphasis in catalysis, sensing and nanomedicine uses.

\section{Non-Covalent and Dynamic Covalent Bonds}

In this section we discuss the two main classes of reversible interactions that can be used for the synthesis of responsive structurally dynamic SCNPs: i) supramolecular (non-covalent) interactions and ii) dynamic covalent bonds. Special emphasis is placed in particular functionalities which are commonly used in the field of supramolecular polymers and dynamic covalent materials.

\subsection{Non-Covalent or Supramolecular Bonds}

Non-covalent interactions, also called supramolecular interactions, can fall into three major classes: i) weak interactions of $0-15 \mathrm{kcal} / \mathrm{mol}$ in bond strength, such as van der Waals forces, hydrophobic interactions, $\pi-\pi$ stacking interactions and hydrogen bonds, ii) medium interactions covering 15-60 kcal/mol in bond strength, such as multiple hydrogen bonds and (weak) metal coordination complexes and iii) strong interactions showing $>60 \mathrm{kcal} / \mathrm{mol}$ in bond strength, such as ionic interactions, host-guest interactions and (robust) metal coordination or chelate complexation. It is worth mentioning that the particular strength of non-covalent bonds depends on external influences such as solvent, temperature, etc. so most non-covalent interactions do not fall into a single category. ${ }^{[17]}$

Selected examples of functional groups involved in weak, medium and strong noncovalent bonds are illustrated in Table 1. Weak non-covalent interactions often involve single 
hydrogen bonding systems like pyridine-benzoic acid, ${ }^{[4]}$ imidazole-carboxylic acid ${ }^{[25]}$ or phenol-pyridine ${ }^{[26]}$ complexes which allow the construction of reversible supramolecular materials. $\pi-\pi$ stacking interactions are other kind of weak reversible forces which have been used with success for the construction of thermally sensitive dynamic polymer networks. ${ }^{\text {[27] }}$ Multiple hydrogen bonds can be classified in two main types: i) self-complementary, in which the hydrogen bonds are formed by dimerization of two identical functional moieties (e.g., ureidopyrimidinone units) $^{[30]}$ and ii) hetero-complementary, in which two different functionalities must be present to establish the hydrogen bonds through self-recognition (e.g., diaminopyridine-thymine HB complexation). ${ }^{[29]}$ Concerning metal complexation, terpyridine ligands are among the most versatile ones allowing the formation of labile complexes with zinc(II) ions, while stable and inert chelates have been demonstrated employing ruthenium(II) and iron(II) ions. $^{[33,34]}$ Host-guest and ionic interactions, although very promising for stabilizing supramolecular materials, ${ }^{[36,37]}$ have been employed less than other non-covalent interactions in supramolecular polymer science. A clear trend in the field is the construction of structurally dynamic materials based on the combination of multiple non-covalent interactions, mainly of two kinds, in an orthogonal way (i.e., by means of selective binding units that do not interfere with each other). ${ }^{[22]}$

\subsection{Dynamic Covalent Bonds}

Table 2 summarizes the most common dynamic covalent bonds used in supramolecular polymer chemistry. As we will see in section 3.2, some of them have been used for the construction of structurally dynamic single-chain nanoparticles, but other dynamic covalent bonds have not been explored for this use yet.

The reversibility and stimuli responsiveness of disulfide bonds have allowed the triggered assembly and disassembly of different polymeric nanostructures, including 
biocompatible and biodegradable nanogel $s^{[38]}$ as well as single-chain nanoparticles, as demonstrated very recently in an elegant paper by Berda and coworkers. ${ }^{[39]}$ Concerning alkoxyamine dynamic covalent bonds, they have been employed for the preparation of a variety of thermoresponsive materials. On exposure to heat $\left(>80{ }^{\circ} \mathrm{C}\right)$ reversible homolytic cleavage of the alkoxyamine moiety occurs through the $\mathrm{C}-\mathrm{O}$ bond yielding a carbon radical and a nitroxide radical. Structurally dynamic covalent polymers containing alkoxyamine moieties both in the main chain and as backbone pendant groups have been demonstrated by Takahara and colleagues. ${ }^{[9,40]}$ Complementary, the thermally reversible Diels-Alder (DA) reaction involves the cycloaddition between an alkene and a diene. The corresponding retroDA reaction usually takes place upon heating to reform the starting materials. Moreover, different dienes and dienophiles can be used to tailor the temperature at which the DA reaction becomes dynamic. In this sense, reversibility of appropriate DA adducts at room temperature has been realized by Lehn and coworkers. ${ }^{[41]}$ Imine $(\mathrm{C}=\mathrm{N})$ formation -by reaction of an amine and an aldehyde- is a reversible reaction which operates under thermodynamic control such as even if kinetically competitive intermediates are initially formed, they are replaced at the end by the thermodynamically most stable products. Using the imine dynamic covalent bond, many complex highly symmetrical molecules and extended structures have been constructed including molecular walkers capable of walking along a track, configurational rotary switches, wholly-organic cages, dynamic catenanes and dynamic rotaxanes, among others. ${ }^{[42]}$ A particular advantage over the hydrolyzable imine bond is the stability of hydrazone $(\mathrm{C}=\mathrm{N}-\mathrm{NH})$ bonds against water and the possibility to perform exchange reactions as well as to break the hydrazone bond in the presence of an acid catalyst. Such advantage has been used by Fulton and coworkers to prepare innovative dynamic covalent single-chain polymeric nanoparticles. ${ }^{[43]}$ Complementary, enamine bond formation has been used to fabricate single-chain nano-objects via "fixed" enamine covalent bonds. ${ }^{[4]}$ Very 
recent results about the reversibility of enamine bonds for efficient nanoparticle disassembly under acidic conditions are summarized in section 3.2.3.

\section{Routes to Reversible Single-Chain Polymer Nanoparticles}

The evolution of the field of single-chain polymeric nanoparticles constructed via supramolecular and dynamic covalent bonds is illustrated schematically in Figure 1. We have distinguished in this figure in blue color the different routes to supramolecular SCNPs, and in red color the pathways to structurally dynamic SCNPs.

Pioneering work by Hawker, Kim and colleagues ${ }^{[24]}$ in 2008 demonstrated an efficient route to supramolecular SCNPs through benzamide dimerization with formation of quadruple hydrogen bonds between benzamide hydrogen bonding motifs. A huge amount of work was later performed by Meijer and coworkers to push forward the field of non-covalent bonded single-chain nanoparticles by introducing two orthogonal techniques based on ureidopyrimidinone (UPy) ${ }^{[45]}$ and benzene-1,3,5-tricarboxamide (BTA) ${ }^{[46]}$ hydrogen bonding motifs. The sequential synthesis of supramolecular nanoparticles involving the complementary self-assembly of UPy and BTA moieties has been recently demonstrated by Meijer's group. ${ }^{[47]}$ Single-chain nanoparticle construction / disassembly based on reversible photo-cross-linking reaction of coumarin activated by UV light at two different wavelengths was reported by Zhao and colleagues in 2011. ${ }^{[48]}$ More recently, host-guest interactions involving cucurbit[n]uril complexation ${ }^{[49]}$ and hydrophobic L-phenylalanine (Phe)-Phe interactions $^{[50]}$ have been employed to synthesize water-born supramolecular single-chain nanoparticles.

Additionally, the seminal work by Fulton and coworkers ${ }^{[43]}$ in 2011 covering the synthesis of SCNPs via dynamic covalent hydrazone bonds paved the way to structurally dynamic unimolecular nanoparticles. A biomimetic approach to reversible polymer nano- 
objects using intramolecular disulfide bond formation has been reported by Berda and colleagues ${ }^{[39]}$ in 2012 . On the other hand, very recent results about acid-triggered disassembly of enamine covalent bonded SCNPs ${ }^{[44]}$ are summarized in section 3.2.3.

\subsection{Single-Chain Polymer Nanoparticles via Non-Covalent Bonds}

\subsubsection{Single-Chain Polymer Nanoparticles via Benzamide Dimerization}

In a pioneering study in the field, Hawker, Kim and colleagues ${ }^{[24]}$ reported the preparation of SCNPs via non-covalent cross-linking of linear chains using benzamide dimerization. Singlechain nanoparticle precursors were prepared by means of reversible addition-fragmentation chain transfer (RAFT) radical copolymerization of methyl methacrylate and a specialty monomer containing a benzamide-dendron that was incorporated into the copolymer at two ratios: 1.5 and $6.1 \mathrm{~mol} \%$. The driving force for the collapse of the individual chains to singlechain nanoparticles was the intramolecular formation of quadruple hydrogen bonds between benzamide hydrogen bonding motifs in apolar solvents at high dilution (see Figure 2). This was accomplished by dissolving the copolymer in a mixture of THF and toluene followed by evaporation of the most volatile THF. Dramatically different behavior was observed for both precursors since large aggregates with diameters of over $100 \mathrm{~nm}$ were observed by using the precursor having only $1.5 \mathrm{~mol} \%$ of benzamide moieties. On the contrary, individual and stable polymer nanoparticles of about $24 \mathrm{~nm}$ in size were formed in toluene at high level of incorporation of the supramolecular units in the copolymer, as determined by scanning force microscopy, dynamic light scattering (DLS) and viscosimetry studies. The possibility to prepare a wide variety of linear single-chain nanoparticle precursors by RAFT copolymerization (with molar mass up to $150 \mathrm{kDa}$ ) and the stimuli-responsiveness of the resulting nanoparticles improves significantly the range of structures, properties and potential 
applications of these reversible unimolecular nano-objects, when compared to covalent bonded single-chain nanoparticles which cannot be reversibly assembled / disassembled.

\subsubsection{Single-Chain Polymer Nanoparticles via Ureido-pyrimidinone Dimerization and} Benzene-tricarboxamide Helical Stacking

Supramolecular polymer nanoparticles were developed by Meijer and coworkers ${ }^{[45]}$ based on polynorbornenes bearing protected 2-ureido-pyrimidinone (UPy) hydrogen bonding motifs (see Figure 3). Nanoparticle formation was followed by size exclusion chromatography (SEC) showing a decrease in "apparent” molecular weight $\left(\mathrm{M}_{\mathrm{w}}\right)$ from $207 \mathrm{kDa}$ to $153 \mathrm{kDa}$ upon UV deprotection for $2 \mathrm{~h}$. It is worth mentioning that the retention time in SEC measurements is inversely proportional to the hydrodynamic size, so a longer nanoparticle vs. precursor SEC retention time (i.e., a lower value of "apparent” $\mathrm{M}_{\mathrm{w}}$ ) is indicative of a more crumpled structure. ${ }^{[51]}$ Interestingly, treatment of the sample with formic acid disrupted the intramolecular hydrogen bonding resulting in an "apparent” $\mathrm{M}_{\mathrm{w}}$ of $193 \mathrm{kDa}$, a value very close to that of the initial precursor. Depending on the solution concentration and due to the dynamic exchange between hydrogen bonds, different aggregation states were found by DLS and atomic force microscopy (AFM) studies: i) single-chain nanoparticles of $16 \mathrm{~nm}$ in size were obtained at high dilution $(<2 \mu \mathrm{g} / \mathrm{mL})$, ii) multi-aggregates of $100-200 \mathrm{~nm}$ in size at moderate dilution $(<10 \mu \mathrm{g} / \mathrm{mL})$, and iii) tendril-like fibrils were observed by AFM measurements on mica substrates upon solvent evaporation from concentrated solutions (>10 $\mu \mathrm{g} / \mathrm{mL})$.

Further extension of the work to poly(methyl methacrylate) (PMMA) precursors bearing protected UPy hydrogen bonding motifs showed a similar morphology evolution as revealed by AFM measurements, although the results were strongly dependent on solvent used, sample concentration and selected surface. ${ }^{[52]}$ Additional experiments indicated that 
slow evaporation rates and the use of solvent-saturated evaporation environments provide the nanoparticles with more mobility during the drying process and, hence, more time for the development of large aggregated structures. ${ }^{[53]}$ In an attempt to understand the interplay between intramolecular supramolecular interactions, polymer rigidity and solvent nature on nanoparticle structure, Meijer and coworkers ${ }^{[54]}$ synthesized different precursors based on polyacrylate, polymethacrylate, polystyrene and polynorbornene backbones of varying stiffness, molecular weight and linking moiety between the backbone and the UPy group but constant percentage of protected UPy groups. On one hand, the flexibility of the polymer backbone, the polymer molecular weight and the nature of the linker were not found to affect single-chain nanoparticle formation. On the other hand, the UPy / solvent interaction was observed to be critical for efficient single-chain nanoparticle formation. As an example, strong intramolecular dimerization was observed in THF without interparticle aggregation whereas both dimerization of the UPy groups and significant interchain interactions were present in $\mathrm{CHCl}_{3}$, as determined by SEC and DLS measurements.

In an effort to introduce orthogonal supramolecular interactions to the UPy noncovalent interactions, Maier and colleagues ${ }^{[46]}$ developed precursors containing the chiral protected benzene-1,3,5-tricarboxamide (BTA) moiety which self-assemble into helical stacks stabilized by tree-fold intermolecular hydrogen bonding upon photo-deprotection (see Figure 4). Nanoparticle formation was followed by circular dichroism (CD) spectroscopy measurements. Interestingly, nanoparticle disassembly was triggered by either an increase in temperature to $80{ }^{\circ} \mathrm{C}$ or addition of an acidic compound like hexafluoroisopropanol (HFIP) which is able to break the hydrogen bonding network between BTA units. The reversibility of the process was demonstrated, respectively, by cooling down slowly to r.t. and by adding a strong base (i.e., quinuclidine) resulting in nanoparticle reconstruction. 
More recently, the possibility to combine UPy and BTA non-covalent interactions in an orthogonal fashion for the synthesis of SCNPs has been demonstrated by Palmans, Meijer and colleagues. ${ }^{[47]}$ One striking result of this work, however, was the coil-like nature of the resulting unimolecular polymer nano-objects in solution after the orthogonal UPy and BTA self-assembly, as undoubtedly deduced from the reported SAXS data which do not display the expected scaling behavior corresponding to globular, highly-folded objects. Conversely, AFM images taken upon solvent removal showed a relatively compact (globular) morphology of the nano-objects in the dry state upon deposition onto mica surfaces. ${ }^{[4]}$ By analogy to recent results reported for covalent bonded single-chain nanoparticles by Pomposo and colleagues ${ }^{[55]}$ the above observations also suggest, in our opinion, the presence of an extended-to-compact transition upon solvent removal that has not been previously appreciated. In this sense, the elongated structure in water at r.t. of poly(oligo(ethylene glycol) methyl ether methacrylate) single-chain nanoparticles synthesized via BTA helical stacking has been recently recognized based on small angle neutron scattering measurements. ${ }^{[56]}$

\subsubsection{Single-Chain Polymer Nanoparticles via Coumarin Photo-dimerization}

The use of the reversible photo-dimerization of coumarin to prepare photo-responsive singlechain nanoparticles has been recently investigated by Zhao and coworkers ${ }^{[48]}$ (see Figure 5). Single-chain nanoparticle precursors were prepared by means of RAFT copolymerization of N,N-dimethylaminoethyl methacrylate (DMAEMA) and a coumarin (CMA) bearing monomer that was incorporated into the copolymer at a level of 7 or $13 \mathrm{~mol} \%$. Well-defined single-chain nanoparticles were obtained by the intrachain photo-dimerization of CMA groups upon $\lambda>310 \mathrm{~nm}$ UV irradiation in a dilute solution of the copolymer in THF (1 $\mathrm{mg} / \mathrm{ml}$ ). After $1 \mathrm{~h}$ of UV-light irradiation, the dimerization degree reached $75 \%$. However, upon changing the UV-light wavelength to $\lambda<260 \mathrm{~nm}$ the reverse photo-cleavage reaction 
occurred only partially since the dimerization degree reduced from $75 \%$ to $38 \%$ after irradiation for $2 \mathrm{~h}$. This partial reversibility was attributed by these authors to the establishment of a photo-stationary state. A detailed investigation about segment mobility as a function of CMA dimerization degree, as determined from the ${ }^{1} \mathrm{H}$ spin-spin relaxation time ( $\left.\mathrm{T}_{2}\right)$ associated to side-chain - $\mathrm{CH}_{2} \mathrm{O}$ - groups, was carried out in $\mathrm{CDCl}_{3}$. Two populations of side-chain segments with different mobilities ( $T_{2}$ values) were observed which were attributed to segments involved in intrachain polymer interactions and those involved in polymersolvent interactions. A clear loss of mobility of the segments involved in intrachain polymer interactions was observed at a CMA dimerization degree of about $36 \%$. Concerning the resulting nanoparticle morphology, different nanoparticle sizes were observed by transmission electron microscopy (TEM) depending on the solvent used during casting. Upon casting from THF, signatures of interparticle aggregation were clearly visible since the nano-objects showed diameters in the range of 30-60 nm which are much larger than those expected for single-chain nanoparticles. After solvent exchange to water through dialysis, nanoparticle diameter was found to be $10-20 \mathrm{~nm}$, as determined by TEM, which was the expected size for SCNPs synthesized from copolymer precursors of $\mathrm{M}_{\mathrm{w}}>100 \mathrm{kDa}$. Interestingly, after dialyzing against water the resulting nanoparticle dispersions were rather stable since no irreversible precipitation was observed after three months.

\subsubsection{Single-Chain Polymer Nanoparticles via Cucurbit[n]uril Complexation}

Inspired by the three-dimensional structures dictated by secondary non-covalent bonds of enzymes that play a crucial role in both molecular recognition and allosteric regulation, Scherman and coworkers ${ }^{[49]}$ introduce a method to obtain reversible single-chain nanoparticles in water through host-guest interactions involving cucurbit[n]uril macrocycles $(\mathrm{CB}[n], n=8$ or 10$)$. $\mathrm{CB}[n]$ s are a family of macrocycle host molecules capable of binding 
two aromatic guest molecules simultaneously (when $n>7$ ). Interestingly, guest binding of $\mathrm{CB}[n]$ macrocycles can be controlled through simple external conditions (e.g., temperature, $\mathrm{pH}$, light, competing guests), thus allowing for reversibility to be easily achieved. Two strategies were followed by these authors to construct water-born single-chain nanoparticles based on $\mathrm{CB}[n]$ complexation.

In a first reported work, ${ }^{[49]}$ ternary complex formation involving $\mathrm{CB}[8]$, viologen (V) and naphtyl (N) functional groups was employed to perform the controlled intramolecular collapse of individual polymer chains under high dilution conditions $(0.1 \mathrm{mg} / \mathrm{mL}$ ) (see Figure 6). Both $\mathrm{V}$ and $\mathrm{N}$ guest functional groups were incorporated in a random fashion onto water soluble poly(N-hydroxyethyl acrylamide) (PHEAM) precursors synthesized via atom transfer radical polymerization (ATRP). The pendant hydroxyl functionality in PHEAM allowed for the conjugation of isocyanate-substituted $\mathrm{V}$ and $\mathrm{N}$ guests for $\mathrm{CB}[8]$ to the polymer backbone at appropriate levels (5 or 15 mol\%). Single-chain nanoparticles were obtained by stirring a diluted solution of the functional polymer chains followed by addition of the cross-linker, $\mathrm{CB}[8]$. Working at a polymer concentration above $0.1 \mathrm{mg} / \mathrm{mL}$ or changing the order of addition resulted in the formation of multi-chain aggregates. The sizes of the resulting nanoparticles as determined by DLS and AFM measurements were found to depend on the molecular weight of the precursor and the amount of cross-linker, $\mathrm{CB}[8]$, allowing for a range of particle sizes to be easily accessed. It is worth of mentioning that nanoparticle formation was exceptionally fast showing an association rate constant of the ratedetermining step around $5 \times 10^{5} \mathrm{M}^{-1} \mathrm{~s}^{-1}$ as well as completely reversible upon addition of competing guest molecules (e.g., 1-adamantylamine).

A second strategy by Scherman and colleagues ${ }^{[57]}$ to construct water-born single-chain nanoparticles relied on the use of the nor-seco-CB[10] macrocycle, a recently discovered macrocyclic host of the extended $\mathrm{CB}[n]$ family which contains two identical cavities capable 
of simultaneously binding one guest each to form 2:1 ternary complexes. In this case, viologen-decorated precursors were employed to form 2:1 ternary complexes with nor-seco$\mathrm{CB}[10]$ and hence to induce the intramolecular collapse of the polymer chains. However, due to the large hydrodynamic size exhibited by the own precursors (e.g., hydrodynamic radius = $95 \mathrm{~nm}$ for a precursor of only $\mathrm{M}_{\mathrm{w}}=350 \mathrm{kDa}$ ), in our opinion, the formation of multi-chain aggregates instead of single chain nano-objects cannot be excluded, even if monomodal distributions were observed by DLS.

\subsubsection{Single-Chain Polymer Nanoparticles via Hydrophobic L-Phenylalanine Interactions}

In a pioneering work in the field of biodegradable responsive unimolecular nanoparticles, the self-association behavior to single-chain nanoparticles of graft copolymers composed of $\operatorname{poly}(\gamma$-glutamic acid) ( $\gamma$-PGA) -as the hydrophilic segment- and L-phenylalanine (Phe) -as the hydrophobic segment- in aqueous solution has been recently investigated by Akashi and coworkers $^{[50]}$ (see Figure 7). $\gamma$-PGA-(Phe) ${ }_{\mathrm{x}}$ graft copolymers with a grafting degree $(x)$ below $50 \%$ were found to be soluble in phosphate buffered saline (PBS, $\mathrm{pH} 7.4$ ). A combination of techniques (NMR, DLS, SLS) were used to determine the size and number of polymer aggregates per particle $\left(\mathrm{N}_{\mathrm{agg}}\right)$ as a function of $x$ in $\gamma$-PGA-(Phe $)_{\mathrm{x}}$ graft copolymers. Interestingly, the size of $\gamma$-PGA-(Phe) ${ }_{12}, \gamma$-PGA-(Phe) ${ }_{27}, \gamma$-PGA-(Phe) ${ }_{35}$ and $\gamma$-PGA-(Phe) $)_{42}$ was found to be about $8-14 \mathrm{~nm}$ as determined by DLS and TEM measurements and the $\mathrm{N}_{\text {agg }}$ was $c a$. 1 suggesting the formation of single-chain nanoparticles in PBS via hydrophobic interactions. To confirm whether if the SCNPs had hydrophobic domains, fluorescence probe experiments using pyrene were performed. The results obtained from the emission and excitation spectra of pyrene demonstrated that single-chain $\gamma$-PGA-(Phe) ${ }_{35}$ and $\gamma$-PGA-(Phe) $)_{42}$ nanoparticles have hydrophobic nanodomains in PBS and that pyrene can be solubilized into these Phe-rich domains. MNR experiments in $\mathrm{D}_{2} \mathrm{O}$ showed that the protons of the 
nanoparticles display restricted motions as a consequence of the presence of a solid-like environment. The presence of carboxyl groups at the nanoparticle surface provided with a negative zeta potential to the nanoparticles dissolved in PBS. Interestingly, nanoparticle size did not change at $\mathrm{pH}$ from 5 to 9 , with $\mathrm{NaCl}$ concentration $(0-0.15 \mathrm{mM})$ at 4,37 , or $60^{\circ} \mathrm{C}$, and in the presence of serum, but multi-aggregate formation was observed at $\mathrm{pH}<4$. The formation of aggregates was due to ionization of the carboxylic groups at the nanoparticle surface. In addition, these single-chain nanoparticles showed high stability against freezedrying, allowing the lyophilized nanoparticles to be easily redispersed without significant changes in size and structure when compared to the initial unimolecular nano-objects.

\subsection{Single-Chain Polymeric Nanoparticles via Dynamic Covalent Bonds}

\subsubsection{Single-Chain Polymer Nanoparticles via Hydrazone Bonds}

In a seminal work by Fulton and coworkers, ${ }^{[43]}$ dynamic covalent bonded unimolecular nanoparticles were prepared by intrachain reaction of aldehyde-decorated macromolecular precursors with bis-hydrazide cross-linkers, leading to the formation of intramolecular dynamic covalent hydrazone bonds in the presence of trifluoroacetic acid (TFA) as a catalyst. The cross-linking density was found to be controlled by the amount of cross-linker used. Quenching of the TFA catalyst by addition of triethylamine prior to nanoparticle isolation was found to be essential for nanoparticle re-dissolution. Interestingly, a facile functionalization of these nano-objects was demonstrated by adorning nanoparticles of low cross-linking density (e.g., 20\%) with a variety of low molecular weight organic hydrazides/alkoxyamines to obtain SCNPs with among the highest density of functionality incorporated within their structure yet reported (i.e., 80\%). Moreover, due to the dynamic nature of the hydrazone bond, several precursors fully adorned with organic residues were collapsed progressively to SCNPs simply through exchange reactions with a bis-hydrazide linker, demonstrating for the first time the 
ability to adapt the architecture of a single polymer chain through dynamic covalent chemistry (see Figure 8).

\subsubsection{Single-Chain Polymer Nanoparticles via Disulfide Bonds}

The fabrication of dynamic covalent bonded SCNPs that can reversibly undergo a coil to particle transition via formation and cleavage of intrachain disulfide bonds has been recently shown by Berda and colleagues. ${ }^{[39]}$ The synthesis strategy adopted was based on the use of poly(norbornene-exo-anhydride) precursors prepared by ring-opening olefin metathesis (ROMP) polymerization and the use of a 4-aminopheyl disulfide cross-linker. The redoxresponsiveness of the disulfide bond was used by these authors to trigger the reversible disassembly/assembly of the single-chain nanoparticles/individual polymer coils under high dilution conditions (1 mg/mL) (see Figure 9). Hence, disassembly of the single-chain nanoparticles was performed by reductive cleavage of the disulfide cross-links with dithiothreitol (DTT) and the process was followed by SEC. The assembly of the individual polymer coils to single-chain nanoparticles was triggered simply by oxidation with $\mathrm{FeCl}_{3}$ in THF solution at high dilution. Nanoparticle size was determined by a combination of techniques including DLS, TEM and SLS. Interestingly, nanoparticles remained stable in solution for weeks based on repeated SEC measurements.

\subsubsection{Single-Chain Polymer Nanoparticles via Enamine Bonds}

In this section we summarize our very recent results about the disassembly -triggered by a strong acid catalyst- of SCNPs prepared via intrachain enamine bonds. ${ }^{[4]}$ It is well known from organic chemistry textbooks that some enamine products formed by condensation of a ketone and an amine can be broken back to their constituents under acidic conditions. In this sense, we selected methyl acetoacetate, 1, and ethanolamine, 2, as low molecular weight 
analogues to follow their condensation to methyl 3-((2-hydroxyethyl)amino)but-2-enoate, 3, via ${ }^{1} \mathrm{H}$ NMR experiments in deuterated water at r.t. Figure 10 shows the presence of clear ${ }^{1} \mathrm{H}$ NMR signals arising from cis- and trans- enamine protons in 3 after $24 \mathrm{~h}$ of reaction time. As expected, upon addition of a strong acid (i.e., phosphoric acid) a complete disappearance of the ${ }^{1} \mathrm{H}$ NMR peaks assigned to cis- and trans- enamine protons is observed after $24 \mathrm{~h}$ as a consequence of enamine bond rupture.

Based on these promising results obtained with simple model compounds, we investigated the possibility of enamine bond rupture in SCNPs synthesized via intrachain enamine bond formation. ${ }^{[4]}$ To our delight, the SEC trace of a THF solution containing enamine bonded SCNPs after treatment for $24 \mathrm{~h}$ with an excess of phosphoric acid (with respect to the enamine content per nanoparticle) was found to be nearly identical to that of the precursor, suggesting a highly-efficient nanoparticle disassembly process (see Figure 11). Fourier transform infrared (FTIR) spectra, as illustrated in Figure 12, supported these SEC results. Hence, upon SCNP formation new infrared vibration bands at 1651 and $1606 \mathrm{~cm}^{-1}$ assigned to enamine bonds were clearly visible in the FTIR spectrum. Upon acid treatment of the enamine bonded unimolecular nanoparticles, these specific infrared vibration bands totally disappeared, in complete agreement with SEC results. The possibility to perform exchange reactions supporting the dynamic nature of the enamine bond, such as those depicted in Table 2, has been recently demonstrated in our laboratory and will be the subject of a forthcoming publication.

\section{Potential Applications of Reversible Single-Chain Polymeric Nanoparticles}

Potential applications of responsive unimolecular nanoparticles depend strongly on the nature of the backbone chain and the reversible interactions used for their construction, since both factors determine nanoparticle solubility, biodegradability, thermal stability, and so on. Table 
3 provides a summary of the main chemical structures of reversible SCNPs reported in the literature, as well as the techniques employed for the preparation of their corresponding linear precursors. Among others, potential applications of reversible SCNPs include catalysis, sensing and nanomedicine uses.

\subsection{Catalysis}

Reversible single-chain polymethacrylate-based nanoparticles synthesized by Zhao and coworkers $^{[48]}$ via coumarin (CMA) photo-dimerization were evaluated as nanoreactors for the synthesis of gold nanoparticles (AuNP) in situ. Interestingly, the intrachain CMA photodimerization degree was found to have a strong effect on the rate of AuNP formation. Hence, the relative rate of AuNP formation in a THF solution containing single-chain nanoparticles with 64 and 27\% CMA photo-dimerization degree was 4 and 2 times faster than that in a THF solution of the precursor. This provides a means to optically control the kinetics of AuNP formation. The whole reduction process of $\mathrm{AuCl}_{4}$ ions in water to AuNPs of 6-9 nm in diameter was finished in 180 min at r.t. which was much faster than the reaction in THF solution due to the more compact size of the SCNPs in this solvent.

Water-soluble single-chain polymethacrylate-based nanoparticles having a catalytically active Ru-containing hydrophobic cavity have been used by Meijer and coworkers $^{[58]}$ for transfer hydrogenation in water. The nanoparticles were synthesized via BTA helical stacking and were stable in the conditions under which catalysis was performed. These SCNPs exploit their hydrophobic interiors to help drive the catalytic cycle. As an example, by using these catalytic nanoparticles and a [substrate] / [Ru] ratio of $200 / 1$ the quantitative reduction of cyclohexanone to cyclohexanol was carried out in $18 \mathrm{~h}$. The turnover frequencies observed by using these BTA self-assembled nanoparticles $\left(10-20 \mathrm{~h}^{-1}\right)$ compared well to those reported for conventional water-soluble Ru-complexes $\left(1-40 \mathrm{~h}^{-1}\right)$. In 
a further extension of this work, ${ }^{[59]}$ L-proline-funcionalizated precursors were transformed to unimolecular nanoparticles via BTA helical stacking and used as efficient catalyst for the aldol reaction between p-nitrobenzaldehyde and cyclohexanone showing high conversion (99 \% after 120 h), high diastereoselectivity (92\%) but moderate enantioselectivity (73\%). One clear advantage of these nanostructured catalysts is that they can be easily recovered from the aqueous phase after separation of the aldol products by filtration and reused several times without additional purification.

\subsection{Sensing}

Flurorescent single-chain polynorbornene-based nanoparticles have been recently reported by Palmans and coworkers ${ }^{[60]}$ based on the intrachain self-assembly in THF / methylcyclohexane $(\mathrm{MCH})$ mixtures of polynorbornene precursors containing bipyridine substituted BTA units (BiPy-BTAs). An increase in green fluorescent intensity at $520 \mathrm{~nm}$ was observed upon decreased solvent polarity and increased degree of BiPy-BTA functionalization. Interestingly, the high propensity of BiPy-BTA to self-assembly intramolecularly was found to avoid the presence of a significant amount of multi-aggregates for precursors having a BiPy-BTA content of $12 \mathrm{~mol} \%$. Due to the affinity of the bipyridine moieties towards metal ions such as copper (II), these single-chain nano-objects were efficient sensors for these metal ions due to strong quenching of nanoparticle fluorescence upon metal binding. However, a significant temperature-dependent response of the sensor nanoparticles was observed as a consequence of the unexpected competition between metal binding and self-assembly involving BiPy-BTA units.

\subsection{Nanomedicine}


It is well-known that disulfide bonds are susceptible to biochemical reductants such as glutathione (GSH), thioredoxin and peroxiredoxin. In this sense, Thayumanavan and coworkers $^{[38]}$ developed biocompatible and biodegradable nanogels with sizes from 16 to 190 nm using disulfide bonds as cross-linkers through a emulsion-free method that promotes the facile encapsulation of hydrophobic guest molecules. The release of entrapped guest molecules induced by GHS was observed to occur at high GSH concentration (10 mM), corresponding to that found inside cells, whereas at low GSH concentration (10 $\mu \mathrm{M})$, as that outside the cell and within the blood plasma, no significant release was found. This approach that allowed cell internalization after decoration of the nanogel nanoparticles with cell penetrating peptides could be also very promising for the case of SCNPs, as those developed recently by Berda and coworkers ${ }^{[39]}$ using disulfide bonds as reversible intrachain crosslinkers.

The preparation of nanoparticles with sizes in between 30 and $200 \mathrm{~nm}$ for the development of vaccine and drug carriers by self-association of hydrophobically modified polyaminoacids was investigated by Akasi and colleagues, ${ }^{[61]}$ which found that the size of the nanoparticles plays a critical role in controlling immune responses. These results motivated the synthesis of hydrophobically self-assembled SCNPs by these authors ${ }^{[50]}$ based on modified poly( $\gamma$-glutamic acid), a naturally occurring, water-soluble, biodegradable, edible, and non-toxic polyamide that is synthesized by several strains of Bacillus.

Recently, thermoresponsive water-soluble dynamic covalent SCNPs that reversibly transform into a hydrogel have been reported by Fulton and coworkers. ${ }^{[62]}$ The triggered gel formation required the simultaneous application of both low $\mathrm{pH}$ and temperature which would be highly convenient for the development of improved drug delivery systems.

\subsection{Other uses}


A significant effect of morphology on viscosity was found by Hawker, Kim and coworkers ${ }^{\text {[24] }}$ for reversible single-chain nanoparticles synthesized through benzamide dimerization by performing measurement in two different solvents: toluene, in which the individual nanoparticles showed reduced viscosities when compared to control polymers lacking the benzamide moiety, and THF, in which no differences between the nanoparticles and the control polymers were observed since this polar solvent suppress intrachain supramolecular interactions. Certainly, viscosity reduction is of great advantage for many industrial applications, especially those related to paints and coatings.

The thermal triggered transformation of a solution processable formulation containing hydrogen bonded single-chain nanoparticles to a high-performance suparmolecular material was reported by Meijer and colleagues. ${ }^{[52]}$ Hence, soluble films of nanoparticles were found to undergo a curing process upon heating above the materials glass transition temperature, reorganizing the material into an insoluble supramolecular network with intermolecular noncovalent cross-links. This feature is of particular interest for the development of self-curing coatings and potentially self-healing surfaces.

Finally, the great potential of dynamic covalent bonds for the development of a new generation of SCNPs whose reversible structures may be "molded" around target template molecules has been claimed by Fulton and coworkers. ${ }^{[43]}$

\section{Conclusion}

We have described in detail the evolution of the recent field of responsive single-chain nanoparticles constructed via supramolecular interactions and dynamic covalent bonds. Although much effort has been devoted in recent years to the development of single-chain nanoparticles that can be reversible assembled / disassembled in response to external environmental changes (e,g,, solvent, UV-light, heat, $\mathrm{pH}$, redox potential) or even can adapt 
their structure though exchange reactions with competing chemical molecules, this topic of research is still in its infancy when compared to the older areas of supramolecular polymers and dynamic covalent materials from which it takes inspiration. Consequently, many demonstrated concepts from the fields of non-covalent interactions and dynamic covalent bonds are still waiting to be adopted by the emerging area of adaptative single-polymer technologies. In this sense, Tables 2 and 3 could serve as useful guides for the design and construction of new multiresponsive SCNPs via non-covalent and dynamic covalent bonds involving orthogonal reversible interactions.

The dynamic nature of the morphology in reversible single-chain polymer nanoparticles, while offering interesting possibilities for developing adaptative materials with external stimuli-dependent properties, also imposes limitations for their precise characterization (e.g. in solution). In our opinion, strong indications about the non-globular nature of SCNPs in solution, synthesized via irreversible or reversible intrachain interactions, are becoming to appear from both SANS and MD simulations, as well as from SAXS results. A significant effort has to be carried out in next years to unravel the complex relationship between external stimuli and dynamic nanoparticle morphologies both in solution and in solid state.

Concerning applications, current envisioned applications of reversible single-chain nanoparticles include mainly catalysis, sensing and nanomedicine uses, although other enduse applications have been also proposed. Due to the novelty of the field and the increasing interest in the added-value behind reversible bonds, a bright future is expected for orthogonally multiresponsive SCNPs which could impact our ordinary lives in a near future. 


\section{Acknowledgements}

We sincerely acknowledge David Fulton for his guidance during the initial experiments about enamine bond reversibility performed by A. S.-S. at the Chemical Nanoscience Laboratory of Newcastle University. Financial support from the projects MAT2012-31088 (MINECO) and IT-654-13 (GV) is acknowledged. A. S.-S. thanks the PhD grant support of Basque Government.

[1] M. Ouchi, N. Badi, J.-F. Lutz, M. Sawamoto, Nat. Chem. 2011, 3, 917.

[2] L. Pauling, The Nature of the Chemical Bond and the Structure of Molecules and Crystals, Oxford University Press, 1939.

[3] J.-M. Lehn, Supramolecular Chemistry, Wiley-VCH, 1995.

[4] T, Kato, J. M. J. Fréchet, Macromolecules 1989, 22, 3818.

[5] X. Yang, F. Hua, K. Yamato, E. Ruckenstein, B. Gong, W. Kim, C. Y. Ryu, Angew. Chem. Int. Ed. 2004, 43, 6471.

[6] E. M. Todd, S. C. Zimmerman, Tetrahedron 2008, 64, 8558.

[7] S. J. Rowan, S. J. Cantrill, G. R. L. Cousins, J. K. M. Sanders, J. F. Stoddart, Angew. Chem. Int. Ed. 2002, 41, 898.

[8] J. Messens, J.-F. Collet. Antioxid. Redox Sign. 2013, 18, 1594.

[9] H. Otsuka, K. Aotani, Y. Higaki, Y. Amamoto, A. Takahara, Macromolecules 2007, 40, 1429.

[10] X. Chen, M. A. Dam, K. Ono, A. Mal, H. Shen, S. R. Nutt, K. Sheran, F. Wudl, Science 2002, 295, 1698.

[11] G. Deng, C. Tang, F. Li, H. Jiang, Y. Chen, Macromolecules 2010, 43, 1191.

[12] P. T. Corbett, J. Leclaire, L. Vial, K. R. West, J.-L. Wietor, J. K. M. Sanders, S. Otto, Chem. Rev. 2006, 106, 3652. 
[13] O. Ramström, J.-M. Lehn, Nat. Rev. Drug Discov. 2002, 1, 26.

[14] B. Levrand, Y. Ruff, J.-M. Lehn, A. Herrmann, Chem. Commun. 2006, 2965.

[15] R. J. Wojtecki, M. A. Meador, S. J. Rowan, Nat. Mater. 2011, 10, 14.

[16] L. Brunsveld , B. J. B. Folmer , E. W. Meijer, R. P. Sijbesma, Chem. Rev. 2001, 101, 4071.

[17] J. M. Pollino, M. Weck, Chem. Soc. Rev. 2005, 34, 193.

[18] T. Maeda, H. Otsuka, A. Takahara, Prog. Polym. Sci. 2009, 34, 581.

[19] M. C. Stuparu, A. Khan, C. J. Hawker, Polym. Chem. 2012, 3, 3033.

[20] A. Bertrand, F. Lortie, J. Bernard, Macromol. Rapid Commun. 2012, 33, 2062.

[21] S.-L. Li, T. Xiao, C. Lin, L. Wang, Chem. Soc. Rev. 2012, 41, 5950.

[22] O. Altintas, C. Barner-Kowollik, Macromol. Rapid Commun. 2012, 33, 958.

[23] A. Sanchez-Sanchez, I. Perez-Baena, J. A. Pomposo, Molecules 2013, 18, 3339.

[24] M. Seo, B. J. Beck, J. M. J. Paulusse, C. J. Hawker, S. Y. Kim, Macromolecules 2008, 41, 6413.

[25] T. Kawakami, T. Kato, Macromolecules 1998, 31, 4475.

[26] A. Ruiz de Luzuriaga, I. Garcia, D. Mecerreyes, A. Etxeberria, J. A. Pomposo, Polymer 2010, 51, 1355.

[27] S. Burattini, H. M. Colquhoun, J. D. Fox, D. Friedmann, B. W. Greenland, P. J. F. Harris, Wayne Hayes, M. E. Mackay, S. J. Rowan, Chem. Commun. 2009, 6717.

[28] T. Kato, M. Nakano, T. Moteki, T. Uryu, S. Ujiie, Macromolecules 1995, 28, 8875.

[29] J. B. Carroll, A. J. Waddon, H. Nakade, V. M. Rotello, Macromolecules 2003, 36, 6289.

[30] F. H. Beijer, R. P. Sijbesma, H. Kooijman, A. L. Spek, E. W. Meijer, J. Am. Chem. Soc. 1998, $120,6761$.

[31] X. Zhao, X.-Z. Wang, X.-K. Jiang, Y.-Q. Chen, Z.-T. Li, G.-J. Chen, J. Am. Chem. Soc. 2003, 125, 15128. 
[32] V. Berl, M. Schmutz, M. J. Krische, R. G. Khoury and J.-M. Lehn, Chem. Eur. J. 2002, 8, 1227.

[33] H. Hofmeier, U. S. Schubert, Chem. Commun. 2005, 2423.

[34] J.-F. Gohy, H. Hofmeier, A. Alexeev, U. S. Schubert, Macromol. Chem. Phys. 2003, 204, 1524.

[35] J. B. Beck, S. J. Rowan, J. Am. Chem. Soc. 2003, 125, 13922.

[36] B. J. Ravoo, J.-C. Jacquier, G. Wenz, Angew. Chem. Int. Ed. 2003, 42, 2066.

[37] J. Huh, H. J. Park, K. H. Kim, K. H. Kim, C. Park, W. H. Jo, Adv. Mat. 2006, 18, 624.

[38] J.-H. Ryu , S. Jiwpanich, R. Chacko , S. Bickerton, S. Thayumanavan, J. Am. Chem. Soc. 2010, 132, 8246.

[39] B. T. Tuten, D. Chao, C. K. Lyon, E. B. Berda, Polym. Chem. 2012, 3, 3068.

[40] Y. Higaki, Y. Ostuka, A. Takahara, Macromolecules 2006, 39, 2121.

[41] P. Reutenauer, E. Buhler, P. J. Boul, S. J. Candau, J. M. Lehn, Chem. Eur. J. 2009, 15, 1893.

[42] M. E. Belowich, J. F. Stoddart, Chem. Soc. Rev. 2012, 41, 2003.

[43] B. S. Murray, D. A. Fulton, Macromolecules 2011, 44, 7242.

[44] L. Buruaga, J. A. Pomposo, Polymers 2011, 3, 1673.

[45] E. J. Foster, E. B. Berda, E. W. Meijer, J. Am. Chem. Soc. 2009, 131, 6964.

[46] T. Mes, R. van der Weegen, A. R. A. Palmans, E. W. Meijer, Angew. Chem. Int. Ed. 2011, 50, 5085.

[47] N. Hosono, M. A. J. Gillissen, Y. Li, S. S. Sheiko, A. R. A. Palmans, E. W. Meijer, J. Am. Chem. Soc. 2013, 135, 501.

[48] J. He, L. Tremblay, S. Lacelle, Y. Zhao, Soft Matter 2011, 7, 2380.

[49] E. A. Appel, J. Dyson, J. del Barrio, Z. Walsh, O. A. Scherman, Angew. Chem. Int. Ed. 2012, 51, 4185. 
[50] T. Akagi, P. Piyapakorn, M. Akashi, Langmuir 2012, 28, 5249.

[51] J. A. Pomposo, I. Perez-Baena, L. Buruaga, A. Alegría, A. J. Moreno, J. Colmenero, Macromolecules 2011, 44, 8644.

[52] E. B. Berda, E. J. Foster, E. W. Meijer, Macromolecules 2010, 43, 1430.

[53] E. J. Foster, E. B. Berda, E. W. Meijer, J. Polym. Sci., Pol. Chem. 2011, 49, 118.

[54] P. J. M. Stals, M. A. J. Gillissen, R. Nicolaÿ, A. R. A. Palmans, E. W. Meijer, Polym. Chem. 2013, 4, 2584.

[55] A. Sanchez-Sanchez, S. Akbari, A. Etxeberria, A. Arbe, U. Gasser, A. J. Moreno, J. Colmenero, J. A. Pomposo, ACS Macro Lett. 2013, 2, 491.

[56] M. A. J. Gillissen, T. Terashima, E. W. Meijer, A. R. A. Palmans, I. K. Voets, Macromolecules 2013, 46, 4120.

[57] E. A. Appel, J. del Barrio, J. Dyson, L. Isaacs, O. A. Scherman, Chem. Sci. 2012, 3, 2278.

[58] T. Terashima , T. Mes , T. F. A. De Greef , M. A. J. Gillissen , P. Besenius , A. R. A. Palmans , E. W. Meijer, J. Am. Chem. Soc. 2011, 133, 4742.

[59] E. Huerta, P. J. M. Stals, E. W. Meijer, A. R. A. Palmans, Angew. Chem. Int. Ed. 2013, 52, 2906.

[60] M. A. J. Gillissen, I. K. Voets, E. W. Meijer, A. R. A. Palmans, Polym. Chem. 2012, 3, 3166.

[61] H. Kim, T. Uto, T. Akagi, M. Baba, M. Akshi, Adv. Funct. Mater. 2010, 20, 3925.

[62] D. E. Whitaker, C. S. Mahon, D. A. Fulton, Angew. Chem. Int. Ed. 2013, 52, 956. 
Table 1. Illustration of non-covalent interactions used in supramolecular polymer chemistry.

\begin{tabular}{|c|c|c|c|}
\hline $\begin{array}{c}\text { Non-Covalent } \\
\text { Interaction } \\
\text { Strength }^{\mathrm{a}}\end{array}$ & $\begin{array}{c}\text { Non-Covalent } \\
\text { Interaction } \\
\text { Type }^{\mathrm{b}}\end{array}$ & Functional Groups Involved & Ref. \\
\hline $\mathrm{W}$ & HB & Pyridine - Benzoic acid & 4 \\
\hline $\mathrm{W}$ & $\mathrm{HB}$ & Imidazole - Carboxylic acid & 25 \\
\hline $\mathrm{W}$ & HB & Phenol - Pyridine & 26 \\
\hline $\mathrm{W}$ & $\pi-\pi$ & Pyrene-Diimide & 27 \\
\hline $\mathrm{M}$ & HB & Diaminopyridine - Benzoic acid & 28 \\
\hline
\end{tabular}




\begin{tabular}{|c|c|c|c|}
\hline $\mathrm{M}$ & HB & Diaminopyridine - Thymine & 29 \\
\hline $\mathrm{S}$ & HB & Ureidopyrimidinone & 30 \\
\hline $\mathrm{S}$ & HB & Ureidopyrimidinone - Naphtyridine & 31 \\
\hline $\mathrm{S}$ & HB & Diaminopyridine - Cyanuric acid & 32 \\
\hline
\end{tabular}




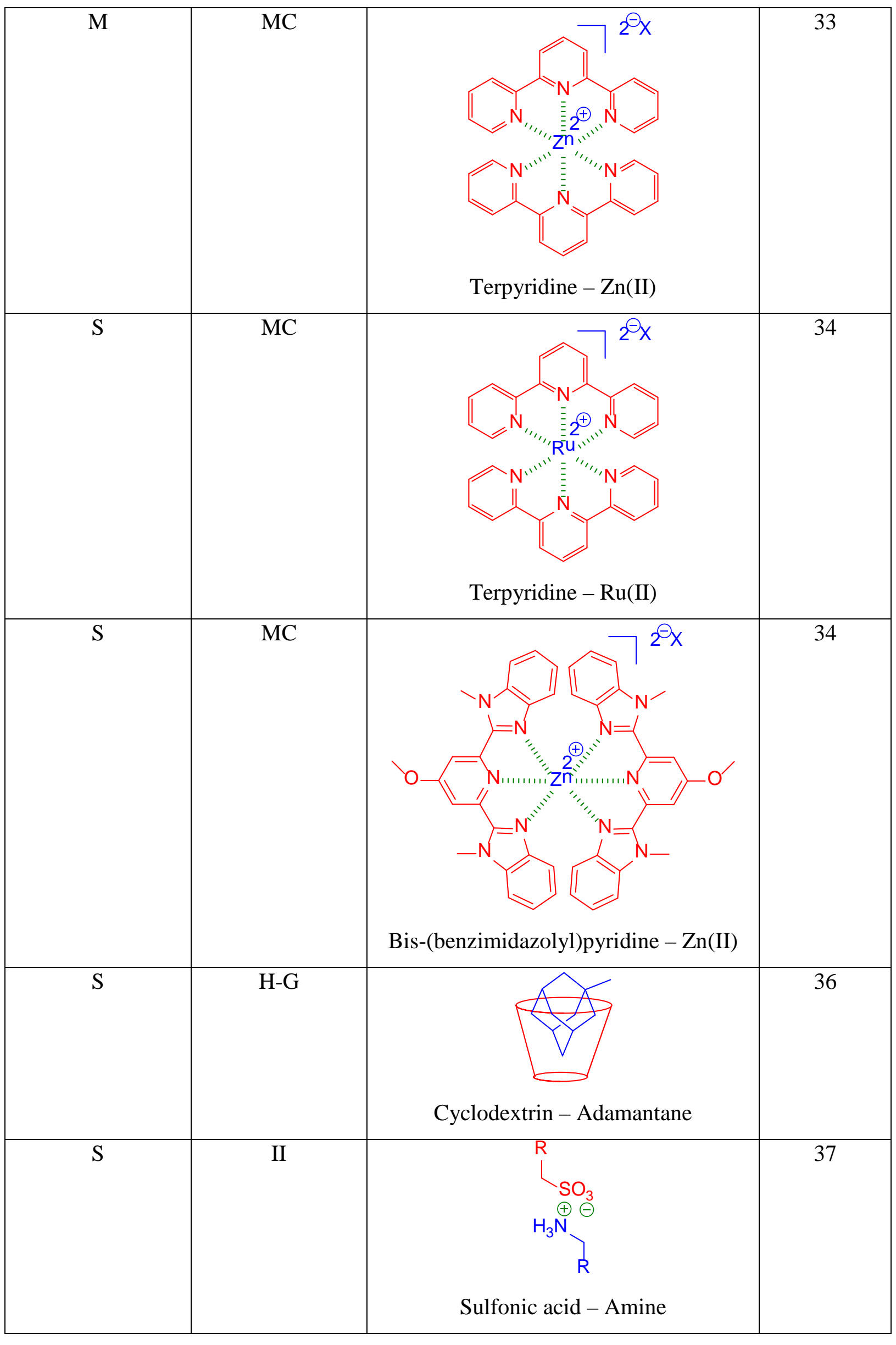


a) $\mathrm{W}=$ Weak. $\mathrm{M}=$ Medium. $\mathrm{S}=$ Strong. ${ }^{\text {b) }} \mathrm{HB}=$ Hydrogen Bonding. $\pi-\pi=\pi-\pi$ interactions. MC $=$ Metal Coordination. II = Ionic Interactions. $\mathrm{H}-\mathrm{G}=$ Host-Guest interactions.

Table 2. Illustration of common dynamic covalent bonds used in supramolecular polymer chemistry.

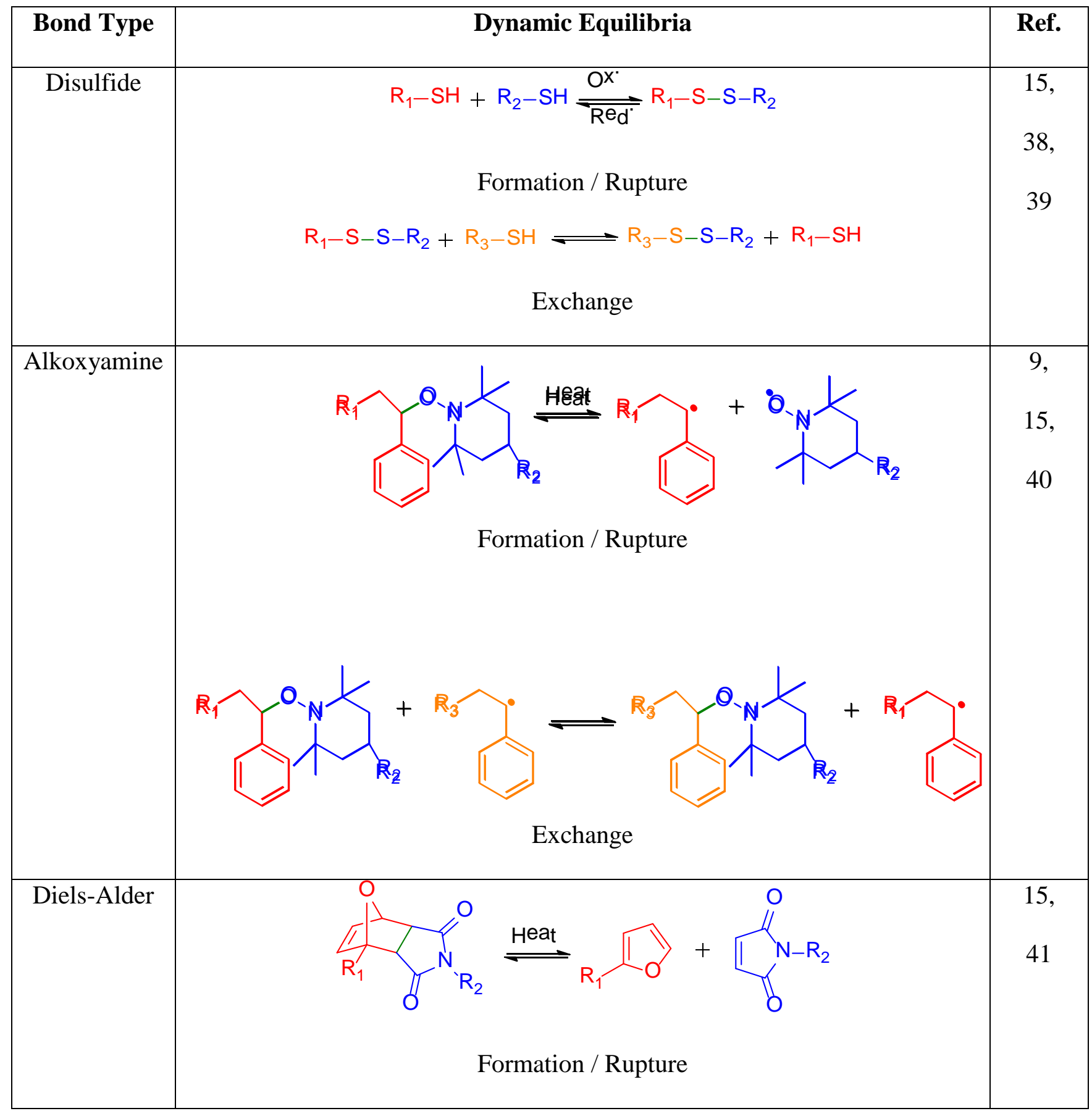




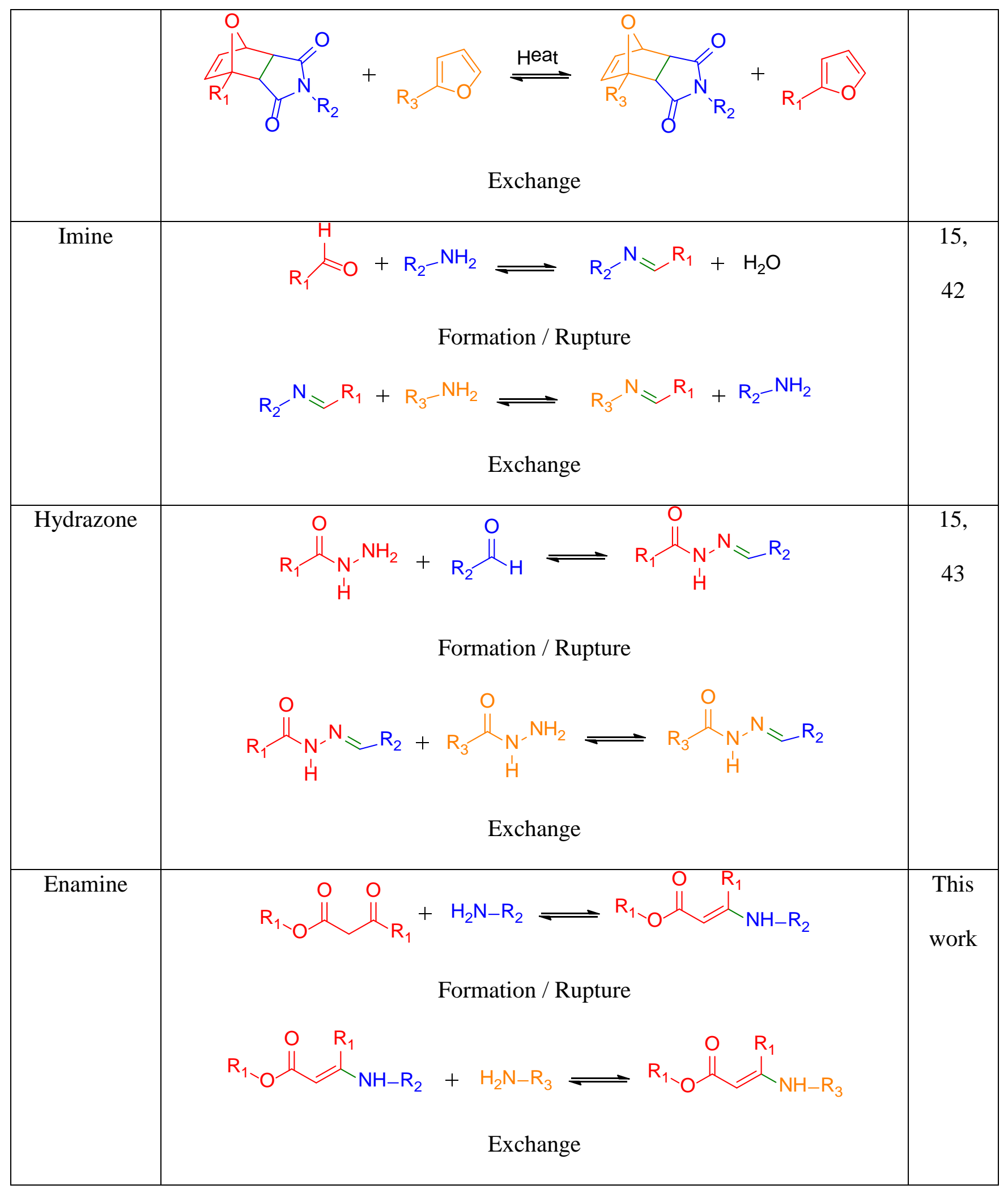


Table 3. Nature of the reversible SCNPs reported in the literature and techniques employed for the synthesis of their linear precursor.

\begin{tabular}{|c|c|}
\hline SCNP nature & Technique used for preparing the linear precursor \\
\hline Poly(meth)acrylates & RAFT, $^{[24,44,48,54]} \mathrm{ATRP}^{[47,52,54,56]}$ \\
\hline Polystyrenes & $\mathrm{NMP}^{[54]}$ \\
\hline Polynorbornenes & ROMP, $^{[39,45,53,54]} \mathrm{ATRP}^{[46]}$ \\
\hline Polyacrylamides & $\mathrm{ATRP}^{[49,57]}$ \\
\hline Polyaminoacids & $\mathrm{PF}^{[50]}$ \\
\hline Polybenzaldehydes & $\mathrm{RAFT}^{[43]}$ \\
\hline
\end{tabular}

${ }^{\mathrm{a}}$ RAFT $=$ Reversible addition-fragmentation chain transfer polymerization; ATRP $=$ Atom transfer radical polymerization; NMP = Nitroxide mediated radical polymerization; ROPM $=$ Ring-opening olefin metathesis polymerization; $\mathrm{PF}=$ Polymer functionalization . 


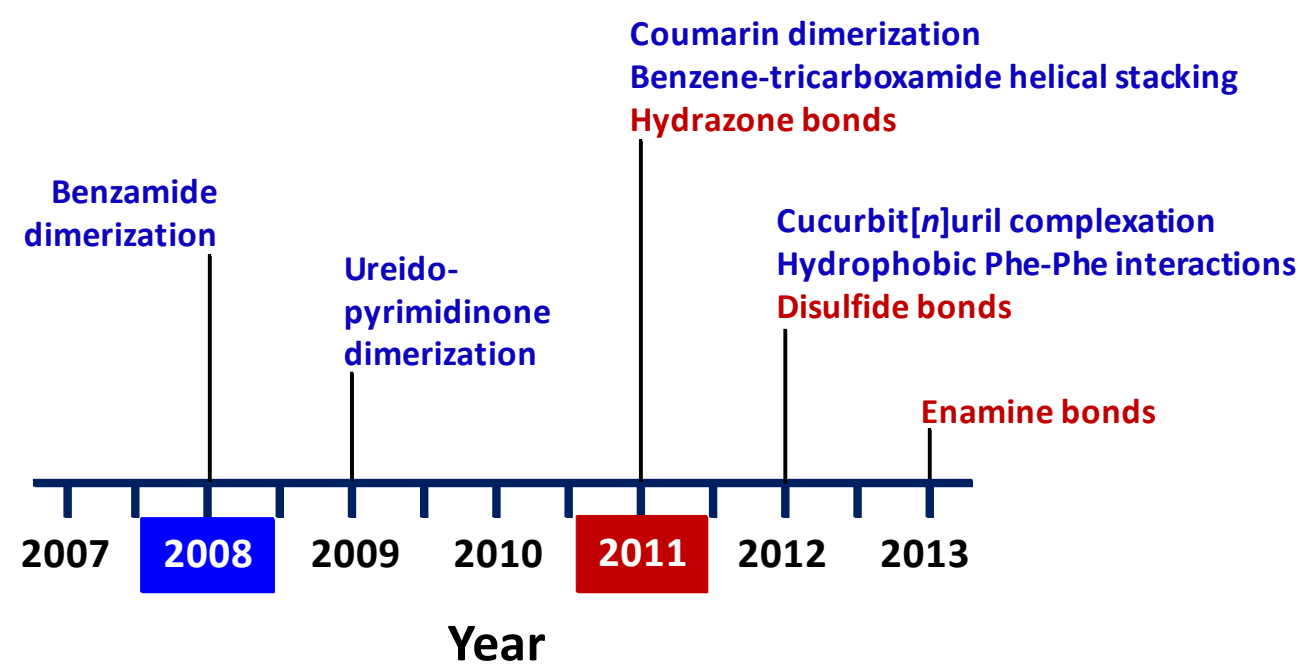

Figure 1. Evolution of the emerging field of reversible SCNPs via non-covalent interactions (blue) and "dynamic" covalent bonds (red). First synthesis of supramolecular SCNPs was carried out by Hawker, Kim and colleagues ${ }^{[24]}$ in 2008. First preparation of dynamic covalent SCNPs was reported by Fulton and coworkers ${ }^{[43]}$ in 2011. 

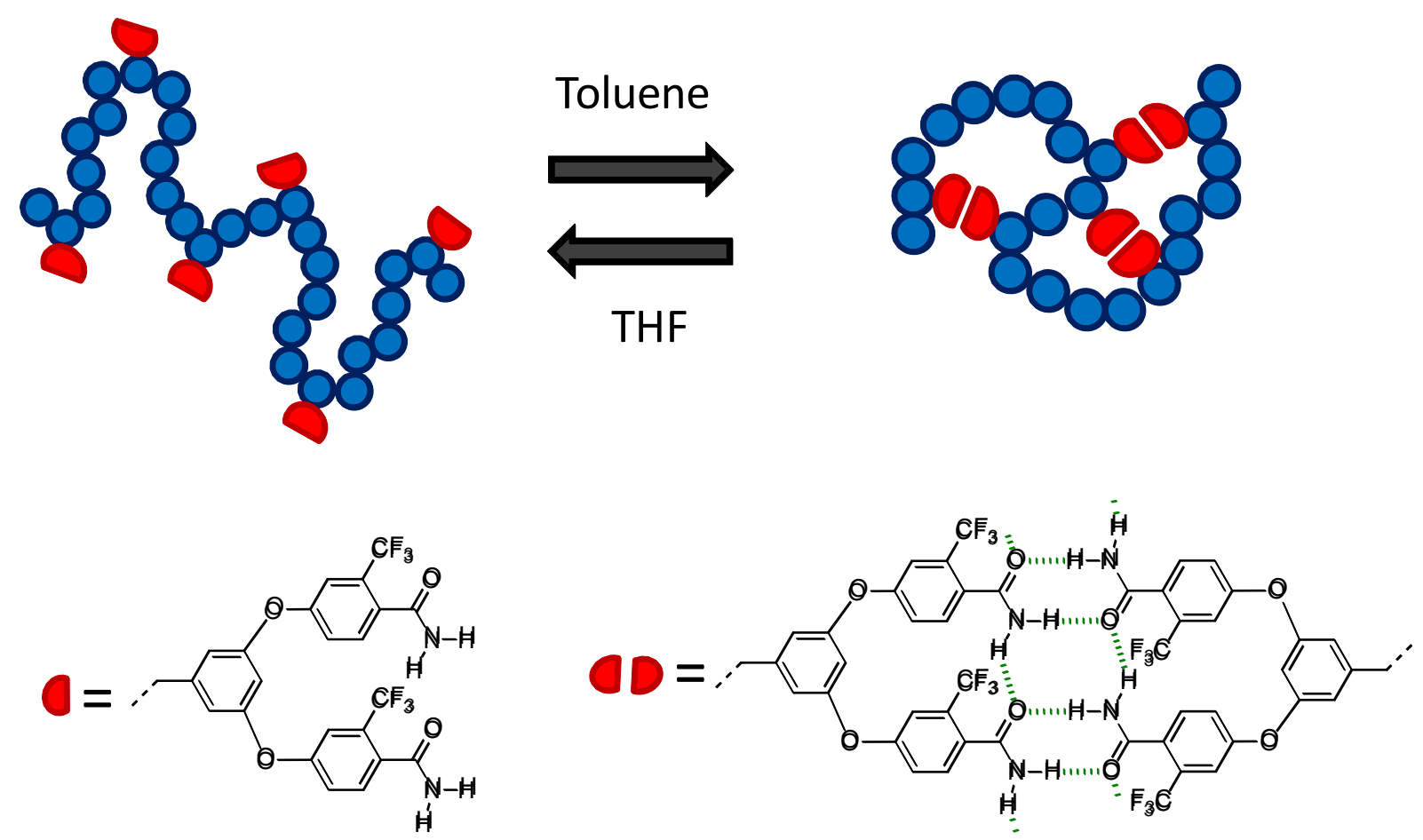

Figure 2. Illustration of the synthesis of reversible SCNPs via intramolecular non-covalent cross-linking of linear precursors containing benzamide moieties in apolar solvents. Higher polarity and hydrogen bonding acceptor characteristics of tetrahydrofuran (THF) trigger nanoparticle disassembly. ${ }^{[24]}$ 

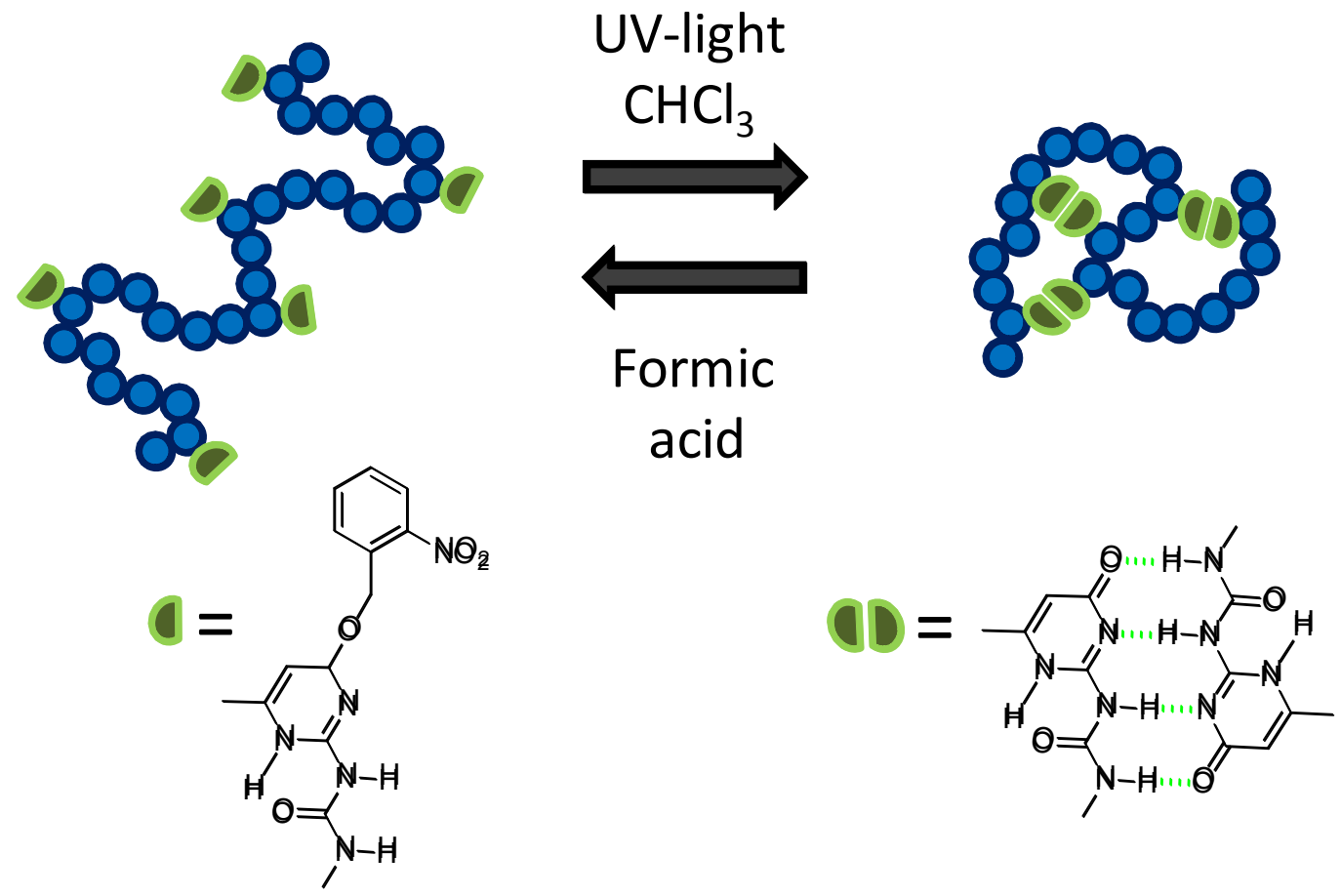

Figure 3. Schematic illustration of supramolecular SCNP formation by UV irradiation of precursors containing protected 2-ureido-pyrimidinone (Upy) groups in chloroform. Disassembly of the non-covalent bonded SCNPs was triggered by formic acid addition. ${ }^{[45]}$ 


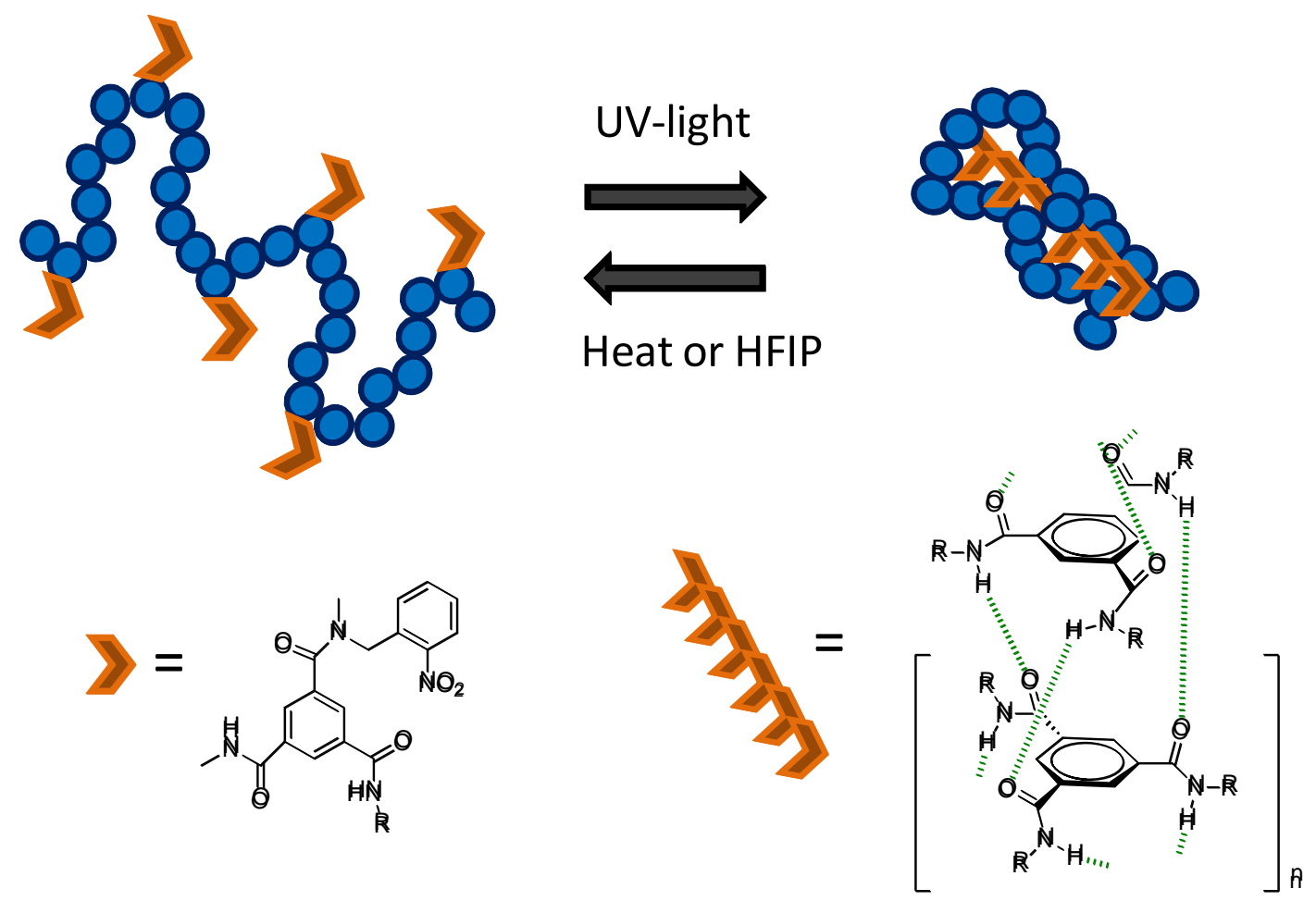

Figure 4. Synthesis of supramolecular SCNPs by UV irradiation of a precursor bearing protected chiral benzene-1,3,5-tricarboxamide (BTA) groups. Disassembly of the noncovalent bonded SCNPs was triggered either by heating above $80{ }^{\circ} \mathrm{C}$ or by addition of hexafluoroisopropanol (HFIP). ${ }^{[46]}$ 


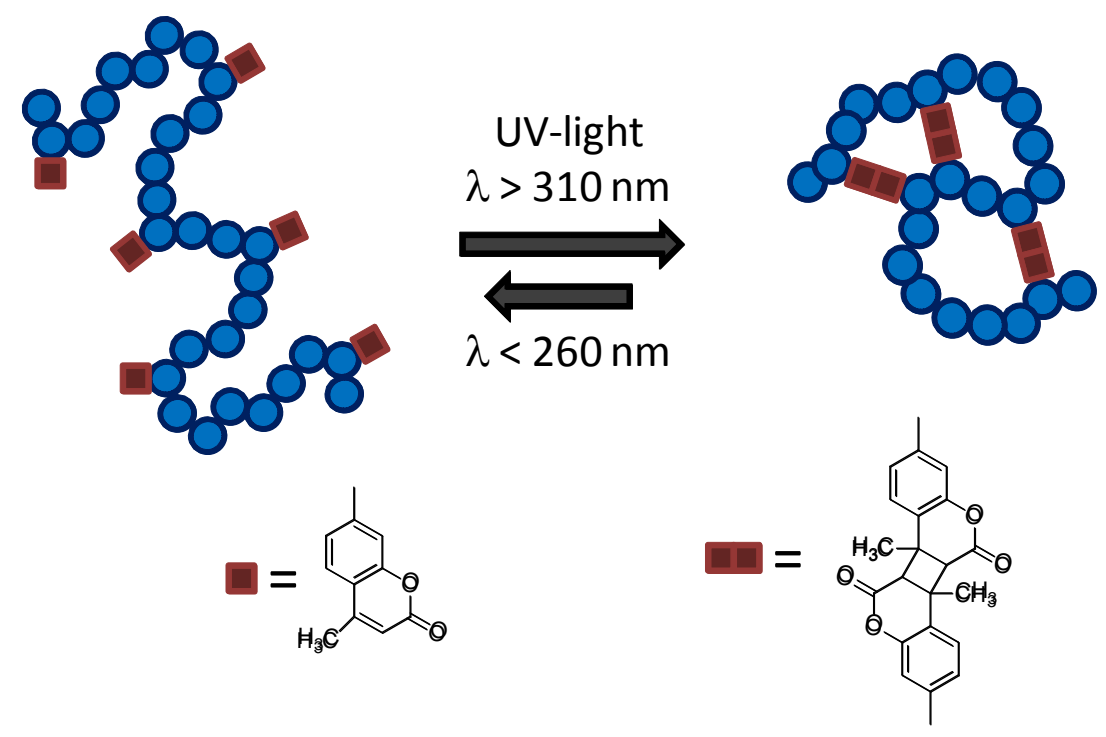

Figure 5. Schematic illustration of the synthesis of SCNPs via coumarin photo-dimerization upon UV irradiation at a wavelength, $\lambda$, above $310 \mathrm{~nm}$. Nanoparticle disassembly was observed upon irradiation at $\lambda<260 \mathrm{~nm}$, although the process was found to be not totally reversible. ${ }^{[48]}$ 


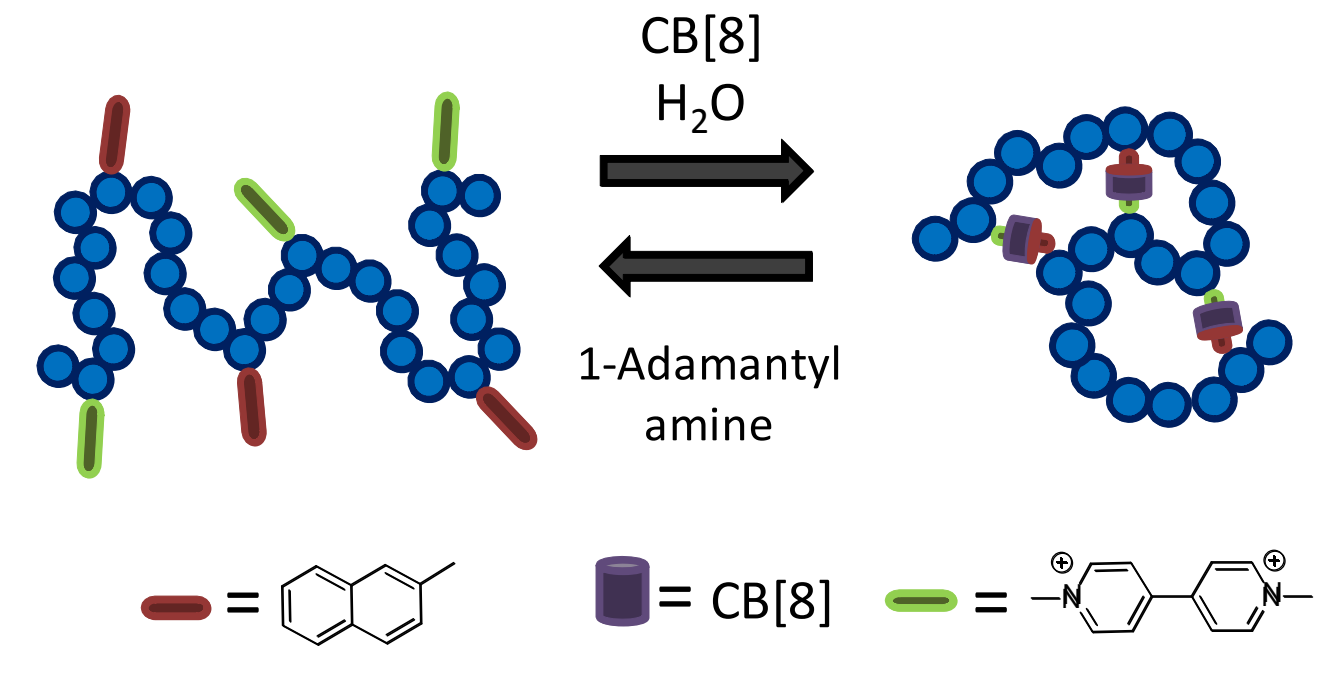

Figure 6. Synthesis of reversible water-borne SCNPs via host-guest interactions between $\mathrm{CB}$ [8] host macrocycle and both viologen and naphtyl guest functional groups. SCNP disassembly was possible by introducing competing guest molecules like 1adamantylamine. $^{[49]}$ 


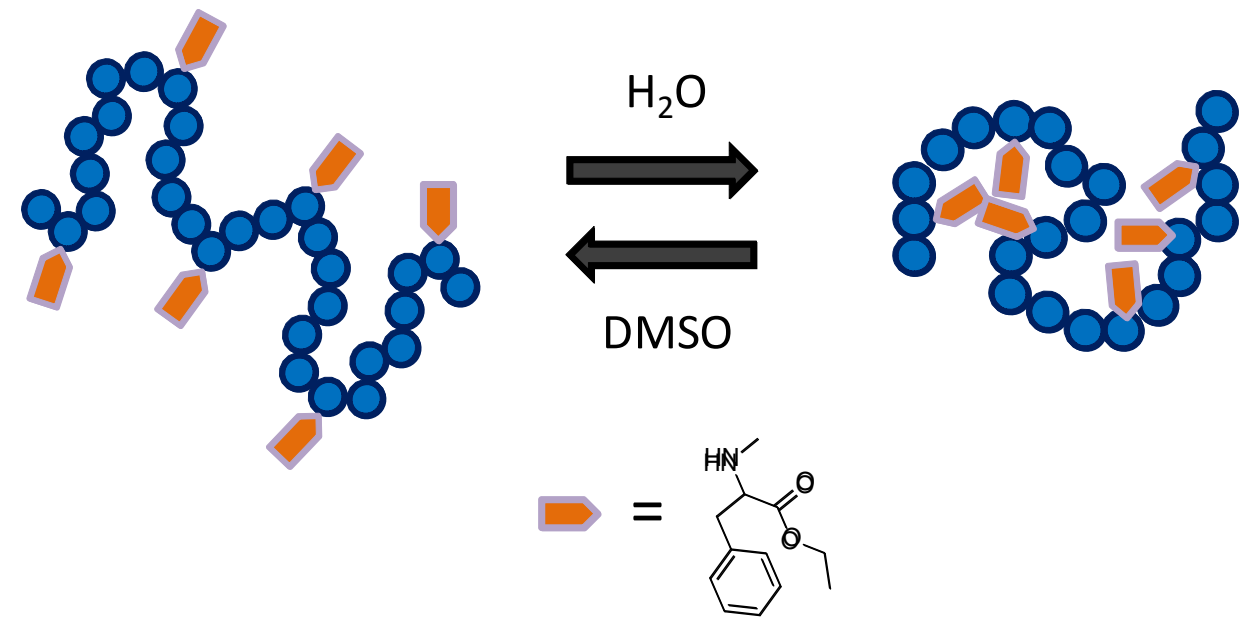

Figure 7. Water-borne reversible SCNPs via hydrophobic interactions involving Lphenylalanine (Phe) segments. SCNPs with a Phe content between 35 and 42 mol\% displayed hydrophobic nanodomains. Disassembly of the SCNPs can be performed by changing from water to organic solvents like dimethyl sulfoxide (DMSO). ${ }^{[50]}$ 

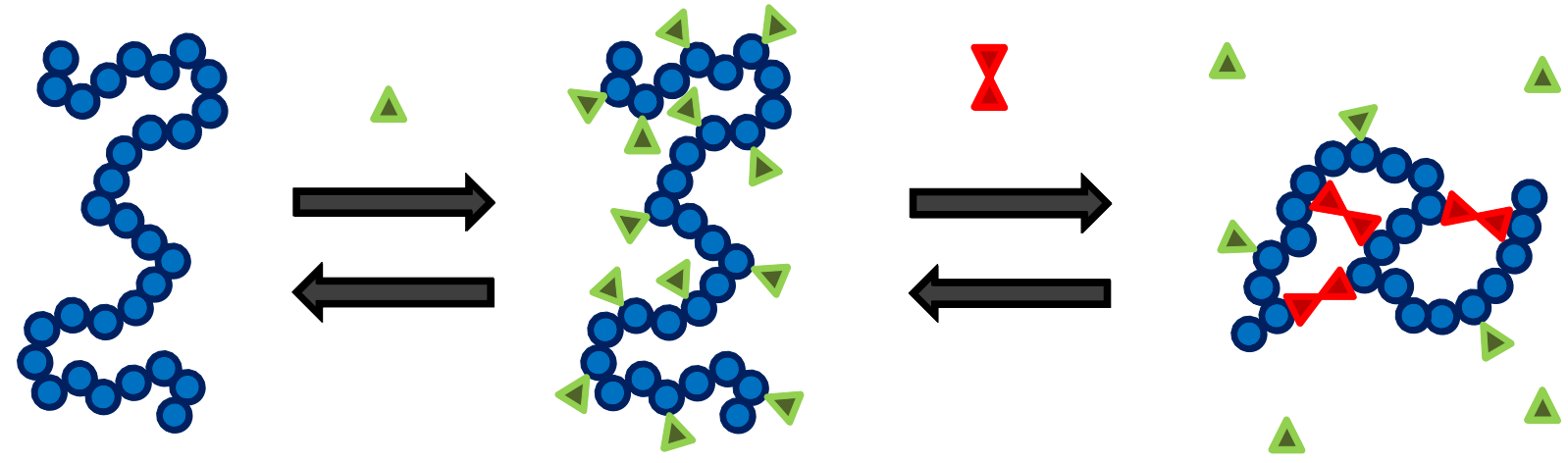

Figure 8. By exploiting the dynamic nature of hydrazone bonds, linear poly(vinylbenzaldehyde) chains were decorated by reaction of their aldehyde units with monoacyl hydrazides (green symbols) and then intramolecularly cross-linked to SCNPs through exchange reactions with a bis-hydrazide cross-linker (green symbols) at high dilution. ${ }^{[43]}$ 


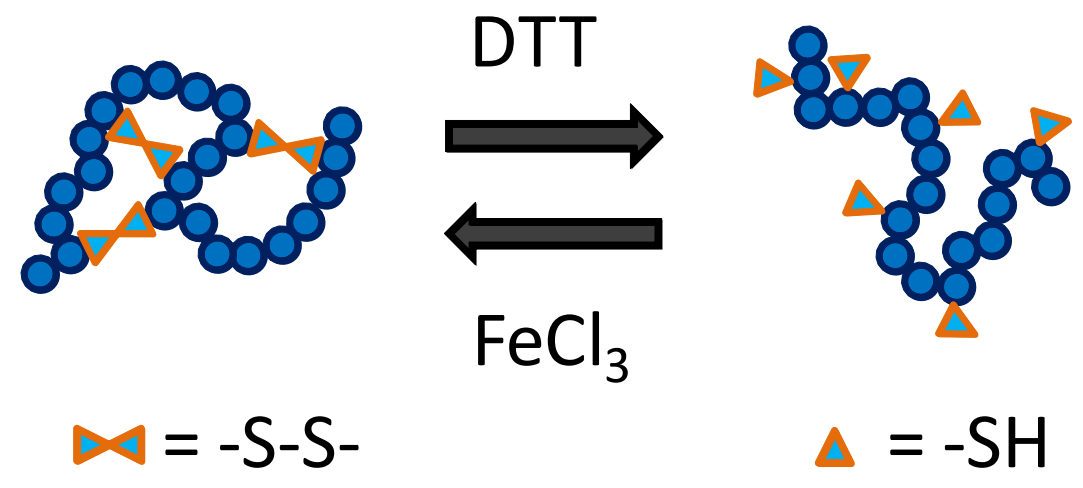

Figure 9. Reversible SCNPs intrachain cross-linked with disulfide bonds can be disassembled to linear chains by reductive cleavage with dithiothreitol (DTT), and re-assembled to unimolecular nanoparticles by oxidation with iron (III) chloride $\left(\mathrm{FeCl}_{3}\right)$ under high dilution conditions. $^{[39]}$ 
A)

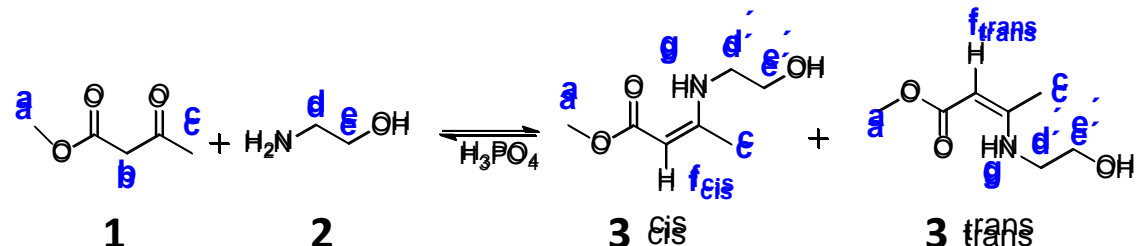

B)

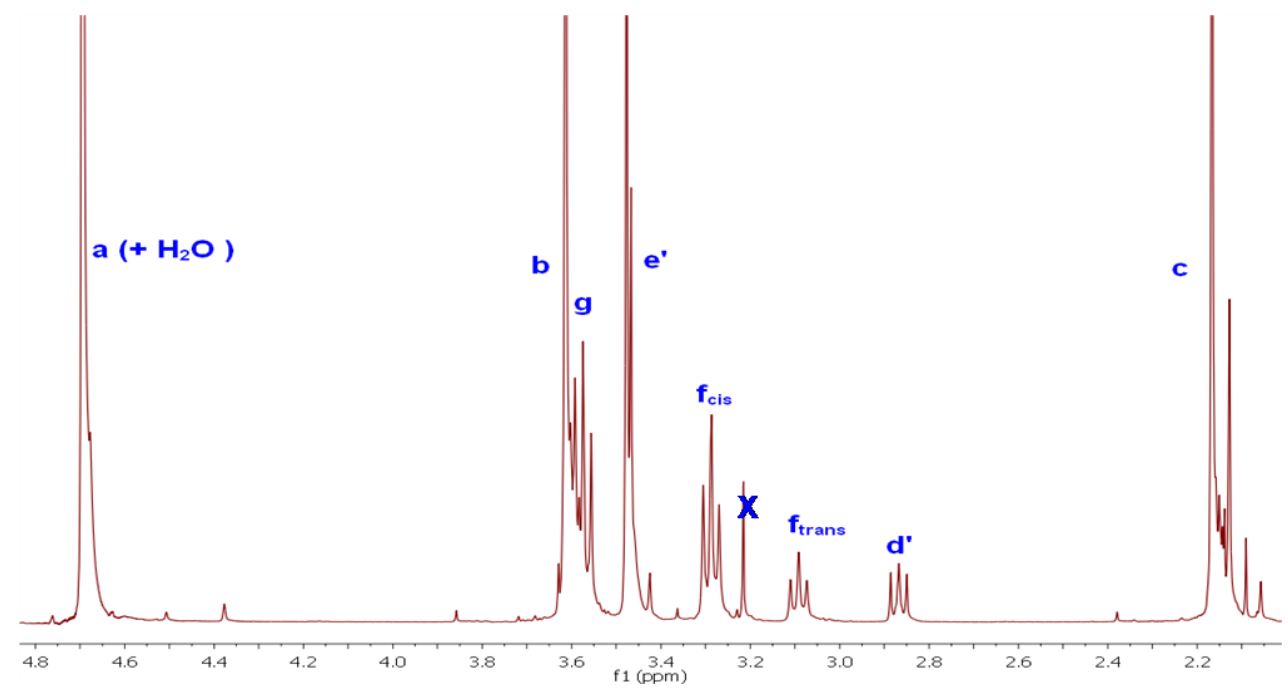

C)

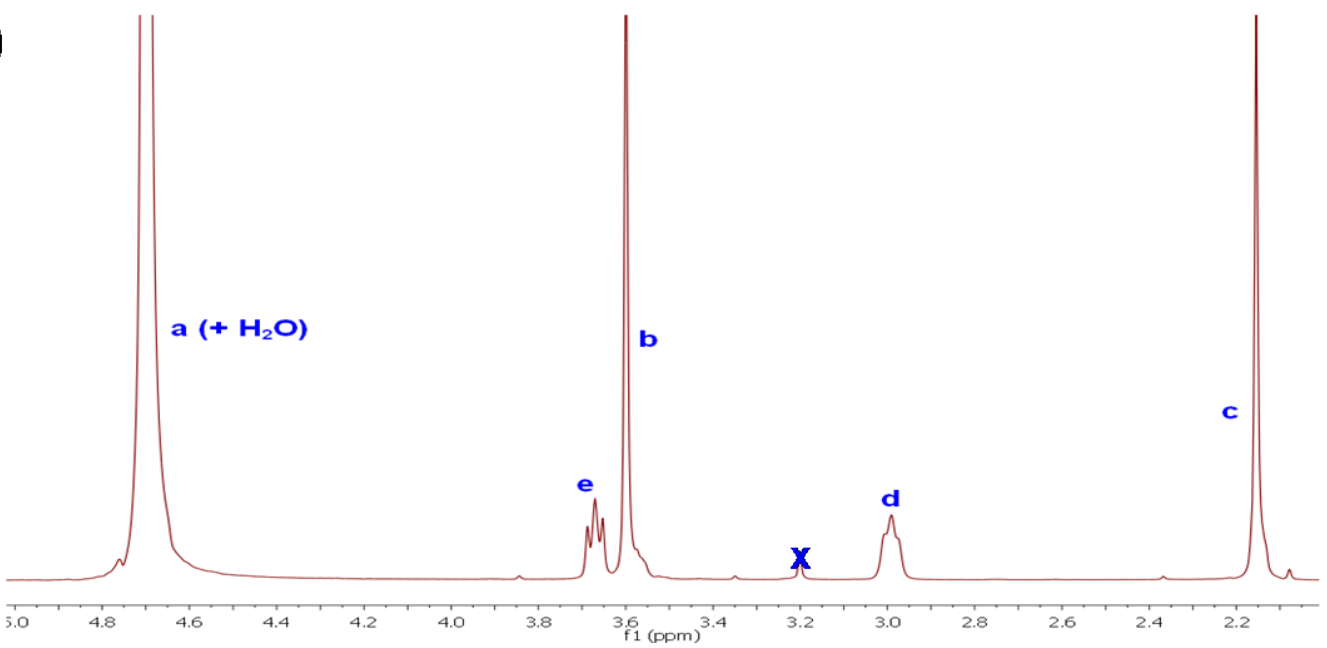

Figure 10. A) Low molecular weight compounds selected for the investigation of enamine bond reversibility under strong acidic conditions and assignation of the corresponding protons for ${ }^{1} \mathrm{H}$ NMR spectroscopy. B) ${ }^{1} \mathrm{H}$ NMR spectrum of 3-((2-hydroxyethyl)amino)but-2-enoate, 3, in $\mathrm{D}_{2} \mathrm{O}$ at r.t. after condensation from methyl acetoacetate, 1, and ethanolamine, 2. C) After reaction of 3 with phosphoric acid (deuterated), no sign of the cis- and trans- enamine protons are visible in the ${ }^{1} \mathrm{H}$ NMR spectrum due to enamine bond rupture. 


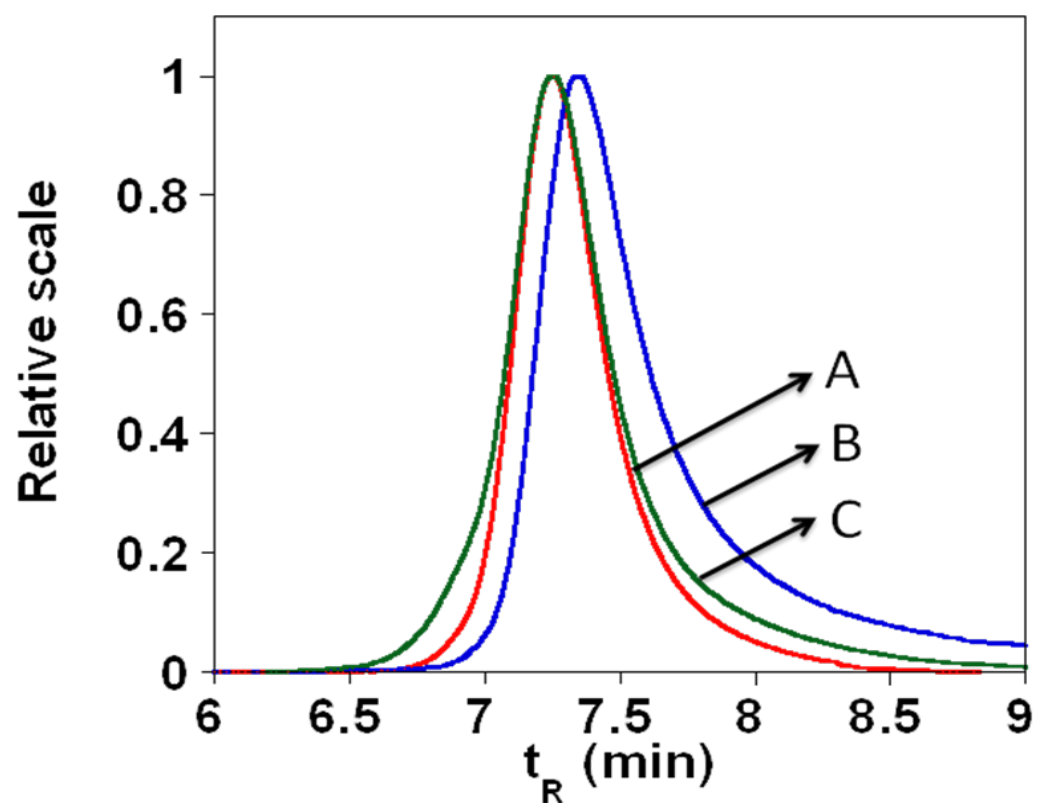

Figure 11. Investigation of enamine bond reversibility in SCNPs: A) SEC trace corresponding to the linear precursor (red curve), B) SEC trace of SCNPs synthesized from the linear precursor via intramolecular enamine bonds ${ }^{[44]}$ (blue curve) and C) SEC trace of SCNPs after treatment with phosphoric acid (green curve), supporting the SCNP disassembly to linear chains. 


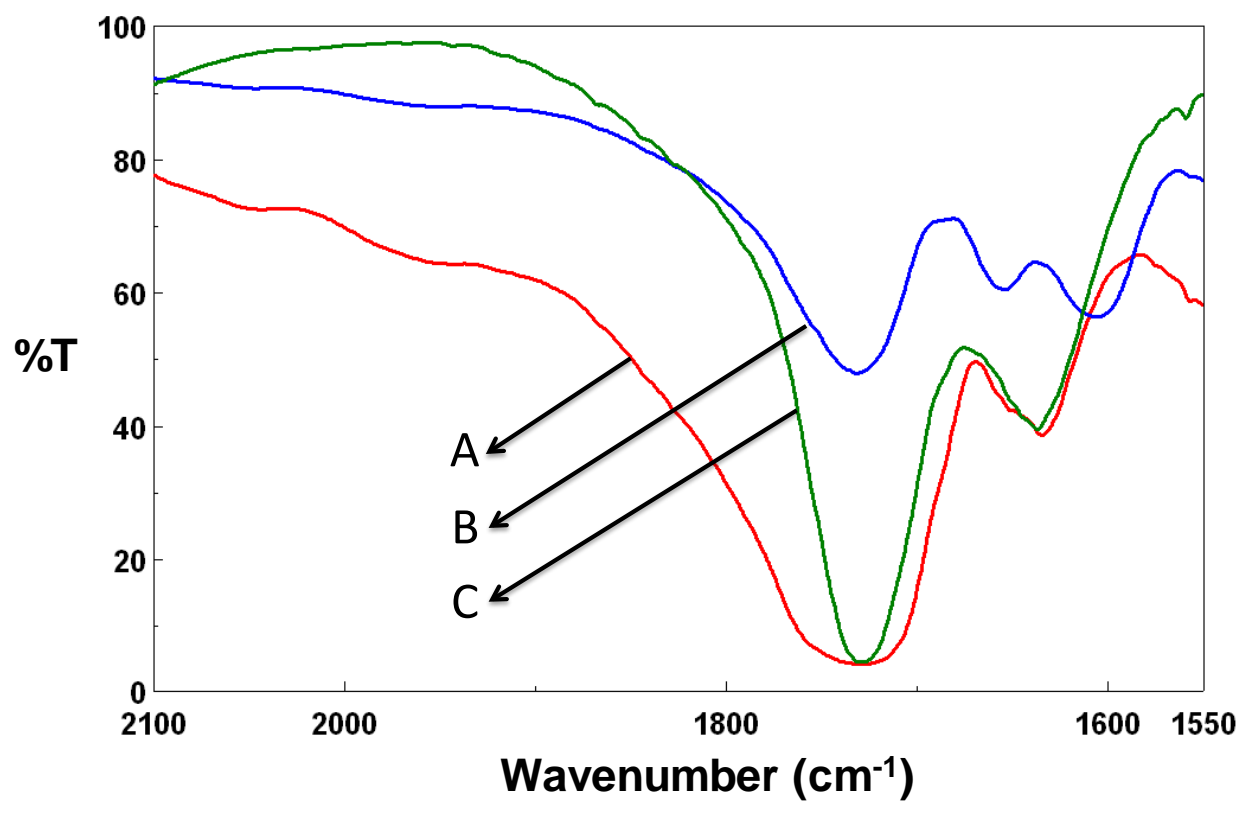

Figure 12. FTIR spectra corresponding to the vibration zone of carbonyl and enamine bonds for: A) single-chain nanoparticle precursor (red curve), B) SCNPs synthesized from the linear precursor via intramolecular enamine bonds ${ }^{[44]}$ (blue curve) and C) SCNPs after treatment with phosphoric acid showing the disappearance of the characteristic enamine vibration bands at 1651 and $1606 \mathrm{~cm}^{-1}$ (green curve). 


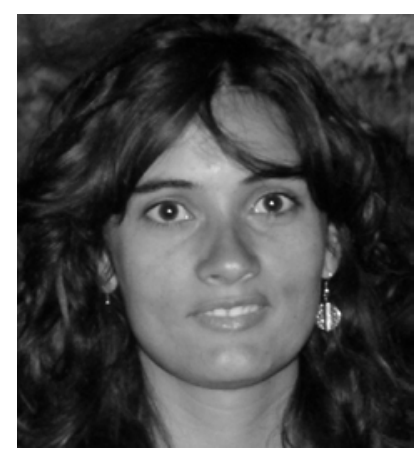

Ana Sanchez-Sanchez received her bachelor degree in Chemistry and master degree in Nanoscience from the University of the Basque Country (UPV/EHU), Spain. She is currently working on her $\mathrm{PhD}$ at the Materials Physics Center (CSIC - UPV/EHU) under the supervision of Prof. José A. Pomposo. Her research focuses on the efficient construction of single-chain polymer nanoparticles mimicking disordered proteins for nanomedicine and chemoselective catalysis applications.

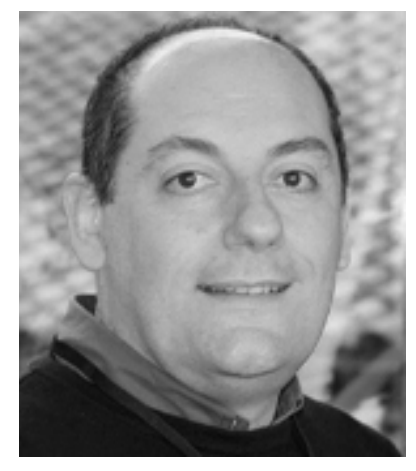

José A. Pomposo received his PhD from the University of the Basque Country in 1994. After more than 15 years of research experience in Materials Science, including the Heading of Cidetec New Materials Department for 12 years, more than 100 scientific publications and 9 international patents, currently, he is Ikerbasque Reseach Professor at the Materials Physics Center, Polymers \& Soft Matter Group, in San Sebastián. His research interests include welldefined uniform soft nano-object synthesis, research in complex single-chain nanoparticle structure, dynamics \& self-assembly, and construction of hybrid organic nanostructures. 


\section{Single-Chain Polymer Nanoparticles via Non-Covalent and Dynamic Covalent Bonds}

Ana Sanchez-Sanchez, José A. Pomposo*

A description of the state of the art in the design, synthesis routes, and applications of reversible single-chain polymer nanoparticles (SCNPs) is provided. After an overview of the main non-covalent interactions and dynamic covalent bonds currently employed in supramolecular polymers and dynamic materials, then we focus on the different reported synthesis approaches and potential applications of (multi)responsive unimolecular soft nanoparticles.

Keywords: Supramolecular; single-chain; nanoparticles; synthesis; self-assembly; catalysis; nanomedicine.

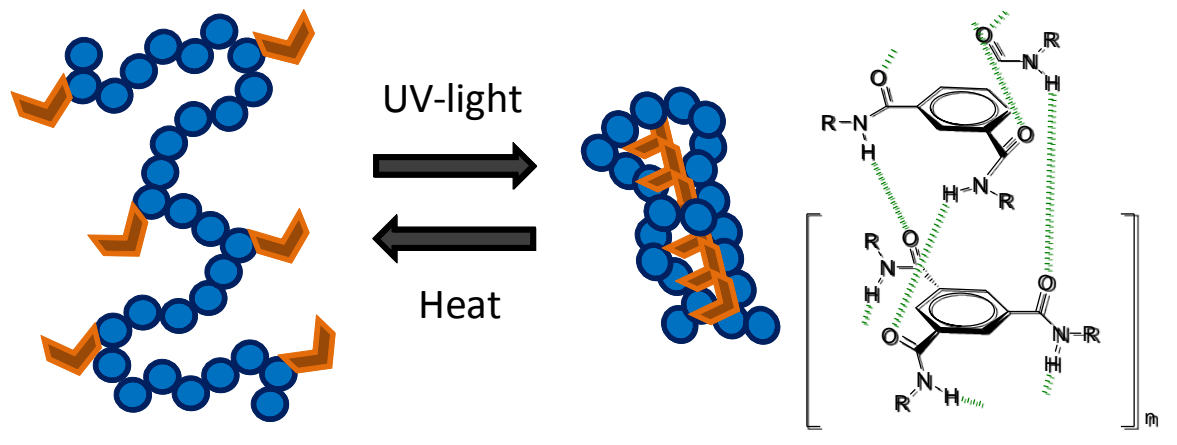

